\title{
Condorcet domains, median graphs and the single-crossing property
}

by Clemens Puppe, Arkadii Slinko

No. 92 | JUNE 2016

\section{WORKING PAPER SERIES IN ECONOMICS}

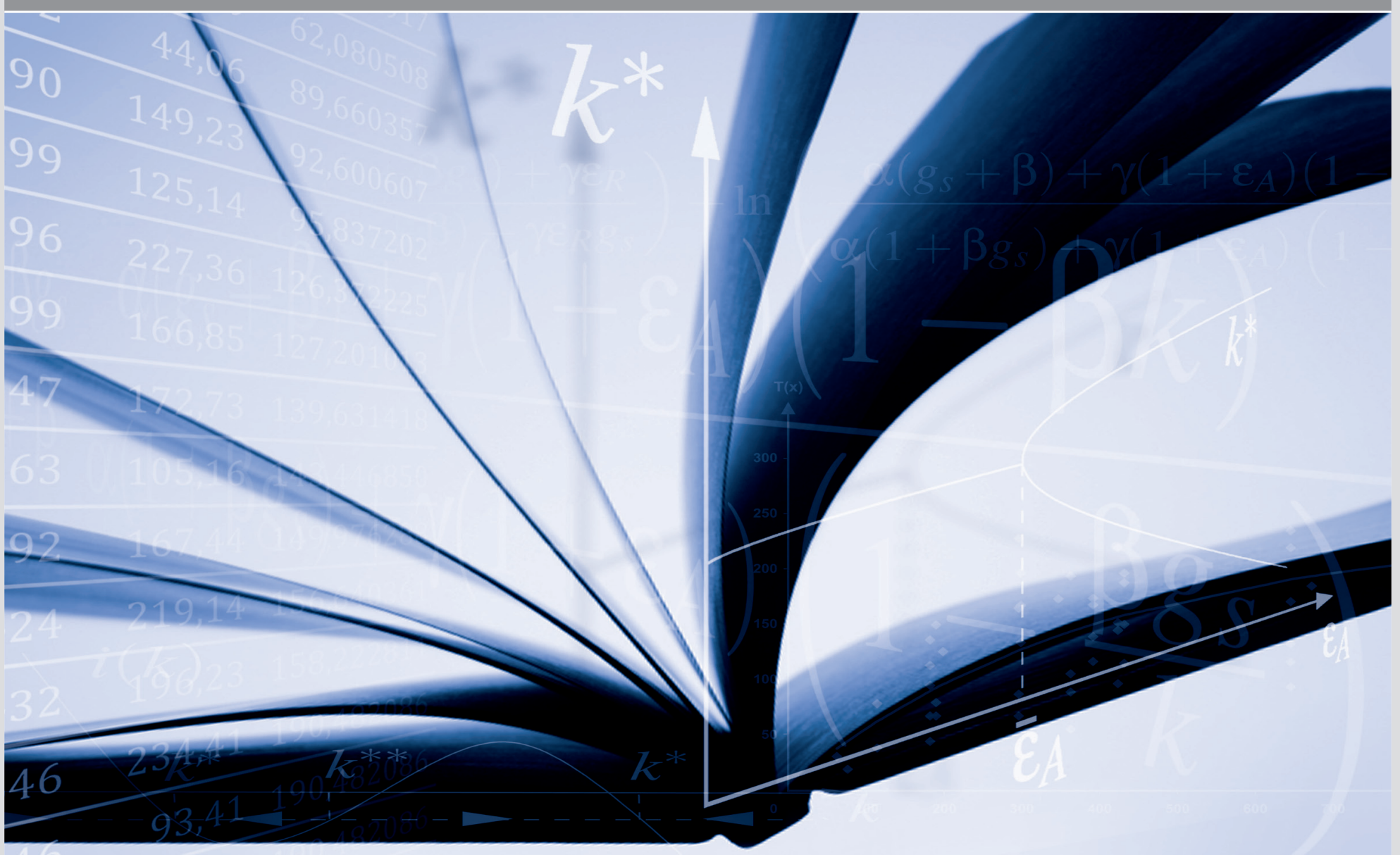




\section{Impressum}

Karlsruher Institut für Technologie (KIT)

Fakultät für Wirtschaftswissenschaften

Institut für Volkswirtschaftslehre (ECON)

Schlossbezirk 12

76131 Karlsruhe

KIT - Die Forschungsuniversität in der Helmholtz-Gemeinschaft

Working Paper Series in Economics

No. 92, June 2016

ISSN 2190-9806

econpapers.wiwi.kit.edu 


\title{
Condorcet Domains, Median Graphs and the Single-Crossing Property*
}

\author{
Clemens Puppe \\ Department of Economics and Management \\ Karlsruhe Institute of Technology (KIT) \\ D - 76131 Karlsruhe, Germany \\ clemens.puppe@kit.edu \\ and \\ Arkadil Slinko \\ Department of Mathematics \\ The University of Auckland \\ Private Bag 92019 \\ Auckland 1142, New Zealand \\ a.slinko@auckland.ac.nz
}

May 2016

\footnotetext{
${ }^{*}$ We are grateful to Dominik Peters for very useful comments on the first version of this paper. Arkadii Slinko was supported by the Marsden Fund grant UOA 1420. More acknowledgements to be added.
} 


\begin{abstract}
Condorcet domains are sets of linear orders with the property that, whenever the preferences of all voters of a society belong to this set, their majority relation has no cycles. We observe that, without loss of generality, every such domain can be assumed to be closed in the sense that it contains the majority relation of every profile with an odd number of voters whose preferences belong to this domain.

We show that every closed Condorcet domain can be endowed with the structure of a median graph and that, conversely, every median graph is associated with a closed Condorcet domain (in general, not uniquely). Condorcet domains that have linear graphs (chains) associated with them are exactly the preference domains with the classical singlecrossing property. As a corollary, we obtain that a domain with the so-called 'representative voter property' is either a single-crossing domain or a very special domain containing exactly four different preference orders whose associated median graph is a 4-cycle.

Maximality of a Condorcet domain imposes additional restrictions on the associated median graph. We prove that among all trees only (some) chains can be associated graphs of maximal Condorcet domains, and we characterize those single-crossing domains which are maximal Condorcet domains.

Finally, using the characterization of Nehring and Puppe [2007] of monotone Arrovian aggregation, our analysis yields a rich class of strategy-proof social choice functions on any closed Condorcet domain.
\end{abstract}

JEL Classification D71, C72

Keywords: Social choice, Condorcet domains, acyclic sets of linear orders, median graphs, single-crossing property, distributive lattice, Arrovian aggregation, strategy-proofness, intermediate preferences. 


\section{Introduction}

The problem of finding and characterizing preference domains on which pairwise majority voting never admits cycles - the so-called Condorcet domains - has a long history in social choice theory. In their seminal contributions, Black [1948] and Arrow [1951] noticed that the domain of all linear preference orders that are single-peaked with respect to some underlying linear spectrum form a Condorcet domain. Later, Sen [1966] provided a characterization of Condorcet domains in terms of the well-known condition of value restriction. Since this early work some progress has been made in understanding the structure of Condorcet domains; see Abello [1991, 2004], Chameni-Nembua [1989], Danilov et al. [2012], Danilov and Koshevoy [2013], Fishburn [1997, 2002], Galambos and Reiner [2008] for important contributions, and Monjardet [2009] for an excellent survey. However, with the exception of Danilov and Koshevoy [2013], the bulk of the results established in the literature pertains only to the special case of connected domains. The analysis of Danilov and Koshevoy [2013], on the other hand, is confined to the case of 'normal' Condorcet domains that contain at least one pair of completely reversed orders.

The present paper provides a unifying general approach by establishing a close connection between Condorcet domains and median graphs (see a comprehensive survey about these in Klavzar and Mulder [1999]) on the one hand, and the well-studied class of singlecrossing domains on the other hand; see Roberts [1977], Gans and Smart [1996], Saporiti [2009], among others.

First, we observe that if a Condorcet domain of linear preference orders admits a profile with an odd number of voters whose (transitive) majority relation does not belong to this domain, then we can add this majority relation to the Condorcet domain and obtain a larger Condorcet domain. We may thus assume without loss of generality that Condorcet domains are closed in the sense that pairwise majority voting among an odd number of individuals always yields an order within the given domain. In particular, all maximal Condorcet domains are necessarily closed.

The concept of betweenness, introduced for linear orders by Kemeny [1959], plays a major role in our analysis. An order is between two orders, or intermediate, if it agrees with all binary comparisons in which the two linear orders agree, (see also Kemeny and Snell [1960], Grandmont [1978]). This allows us to associate a graph to any domain of linear orders by calling two linear orders of this domain neighbors if there are no other linear orders in this domain that are between them.

We show that (i) every closed Condorcet domain on a finite set of alternatives equipped with the neighborhood relation is isomorphic to a median graph, and (ii) for every finite median graph there exists a set of alternatives and a closed Condorcet domain whose graph is isomorphic to the given median graph. Importantly, the median graph corresponding to a Condorcet domain is in general not a subgraph of the permutohedron, which is the graph associated with the universal domain of all strict linear orders (see Section 3 below for detailed explanation $)^{1}$.

\footnotetext{
${ }^{1}$ The permutohedron was defined in Guilbaud and Rosenstiehl [1963] as a polytope. It can also be viewed as a graph in which two vertices are neighbors if they are joined by an edge (which is the 1-skeleton of that polytope). Monjardet [2009] calls it permutoèdre graph.
} 
Our analysis is related to prior work by Nehring and Puppe [2007] and Demange [2012]. Nehring and Puppe [2007] introduce a general notion of a median space and demonstrate its usefulness in aggregation theory; we comment on the relation to their work in more detail below. Demange [2012] shows that, if it is possible to assign linear orders of a certain domain to the vertices of a median graph in such a way that intermediate orders lie on a shortest path in the graph, then the majority relation of any profile with preferences from this domain admits no cycles. However, Demange [2012] takes both the median graph and the preference profile as given and does not address the issue under which conditions the required construction can indeed be carried out. Part (i) of our aforementioned result shows that, in fact, every closed Condorcet domain (with its respective neighborhood relation) is isomorphic to a median graph. The analysis of the present paper thus demonstrates that Demange's requirement of the existence of a median graph is not only sufficient but also necessary for a domain of preferences to admit an acyclic majority relation for all profiles.

The close connection between Condorcet domains and median graphs established here allows one to apply results from the theory of median graphs to shed further light on the structure of Condorcet domains. For instance, as a simple corollary of our analysis we obtain that all closed Condorcet domains that contain two completely reversed linear orders are distributive lattices. This important fact was first established for connected maximal Condorcet domains by Chameni-Nembua [1989] and Abello [1991], and then for all maximal Condorcet domains by Danilov and Koshevoy [2013] who used a different technique. Here we show this for the much larger class of all closed (but not necessarily maximal) Condorcet domains.

While all median graphs give rise to closed Condorcet domains, not all of them correspond to a maximal Condorcet domain. It turns out that in fact certain types of median graphs never enable maximality of the respective Condorcet domains. In particular, we prove that among all trees only (some) chains are associated with maximal Condorcet domains.

Condorcet domains, whose median graphs are chains, have been studied quite extensively in economics under the name of single-crossing domains. They are characterized by the single-crossing property which stipulates that the orders of the domain can be arranged in a chain so that, for any ordered pair of alternatives, the set of all orders that rank one alternative strictly above the other form an interval in this chain. It is wellknown that single-crossing domains have the representative voter property (cf. Rothstein [1991]), i.e., in any profile with an odd number of voters whose preferences belong to the given domain there is one voter whose preference order coincides with the majority relation. We show here that the representative voter property essentially characterizes the class of single-crossing domains. ${ }^{2}$

A maximal single-crossing domain must obviously contain two completely reversed orders. Interestingly, not all maximal single-crossing domains are maximal Condorcet domains, i.e., typically it is possible to add further preference orders to a maximal single-

\footnotetext{
${ }^{2}$ The only exceptions are the domains with exactly four elements such that the associated graph is a 4-cycle; these also satisfy the representative voter property.
} 
crossing domain without generating cycles in the majority relation of any profile. Here, we provide a simple necessary and sufficient condition of when a maximal single-crossing domain is also a maximal Condorcet domain. The condition requires that the 'switched' pairs of alternatives associated with any two consecutive orders of the domain have one element in common.

Our analysis immediately implies that the closed Condorcet domains are exactly the median stable subsets of the space of all linear orders on a given set of alternatives. It thus follows from the results of Nehring and Puppe [2007, 2010] that closed Condorcet domains not only enable pairwise majority voting as a consistent aggregation method but in fact admit a wide range of further aggregation rules satisfying Arrow's independence condition. We adapt their characterization of all monotone Arrovian aggregators to the case of Condorcet domains of linear orders and show that every monotone Arrovian aggregator on a closed Condorcet domain induces a strategy-proof social choice function on the same domain. For each Condorcet domain, we thus obtain a rich class of strategy-proof voting rules, of which the rules identified by Saporiti [2009] for single-crossing domains are special cases.

The remainder of the paper is organized as follows. In the following Section 2, we introduce the concept of a Condorcet domain and observe some of its fundamental properties. In particular, we show that closed Condorcet domains are exactly the median stable subsets of the space of all linear preference orders on a given set of alternatives. Section 3 introduces median graphs and states our main result establishing the correspondence between closed Condorcet domains and median graphs. Section 4 provides the characterization of single-crossing domains, and discusses a weaker version of the singlecrossing property, namely single-crossingness on trees. Section 5 addresses maximality of Condorcet domains, an issue that has already received attention in the literature (cf. Monjardet [2009]). In particular, we prove that trees different from chains are never associated with maximal Condorcet domains, and we characterize the chains (i.e. single-crossing domains) that correspond to maximal Condorcet domains. Any maximal single-crossing domain necessarily contains two completely reversed orders; this does not hold for all maximal Condorcet domains as we show by means of an example. In Section 6, we adapt the characterization of all monotone Arrovian aggregators obtained in Nehring and Puppe [2007] to the case of (closed) Condorcet domains and show how it induces a large class of strategy-proof social choice functions on any such domain. An appendix briefly reviews the theory of geometric interval operators (see, e.g. van de Vel [1993]) and contains the proof of the central Lemma 3.1 using the so-called triangle condition introduced by Bandelt and Chepoi [1996].

\section{Closed Condorcet Domains as the Median Stable Subsets of the Universal Domain}

In this section, we show that the class of domains that are closed under taking the majority relation for all profiles with an odd number of voters are precisely the median stable subsets of the space of linear orders endowed with the Kemeny betweenness relation. 
Most of the results in this section are not original. In Theorem 1 we gathered a number of classical characterizations of Condorcet domains and Theorem 2 can be derived from the analysis in Nehring and Puppe [2007] who investigated a more general class of median spaces. Nevertheless, we believe that our exposition and the short proof of the fundamental characterization result, Theorem 2 below, will help to clarify and unify several different approaches in the literature.

\subsection{Condorcet Domains}

Consider a finite set of alternatives $X$ and the set $\mathcal{R}(X)$ of all (strict) linear orders (i.e., complete, transitive and antisymmetric binary relations) on $X$ which we will refer to as the universal domain. Any subset $\mathcal{D} \subseteq \mathcal{R}(X)$ will be called a domain of preferences or simply a domain. A profile $\rho=\left(R_{1}, \ldots, R_{n}\right)$ over $\mathcal{D}$ is an element of the Cartesian product $\mathcal{D}^{n}$ for some number $n \in \mathbb{N}$ of 'voters', where the linear order $R_{i}$ represents the preferences of the $i$ th voter over the alternatives from $X$. A profile with an odd number of voters will simply be referred to as an odd profile. Frequently, we will denote linear orders simply by listing the alternatives in the order of decreasing preference, e.g., a linear order that ranks $a$ first, $b$ second, $c$ third, etc., is denoted by $a b c \ldots$.

The majority relation associated with a profile $\rho$ is the binary relation $P_{\rho}^{\text {maj }}$ on $X$ such that $x P_{\rho}^{\text {majy }} y$ if and only if more than half of the voters rank $x$ above $y$. Note that, according to this definition, the majority relation is asymmetric and, for any odd profile $\rho$ and any two distinct alternatives $x, y \in X$, we have either $x P_{\rho}^{\text {maj }} y$ or $y P_{\rho}^{\text {maj }} x$. An asymmetric binary relation $P$ is acyclic if there does not exist a subset $\left\{x_{1}, \ldots, x_{m}\right\} \subseteq X$ such that $x_{1} P x_{2}, x_{2} P x_{3}, \ldots, x_{m-1} P x_{m}$ and $x_{m} P x_{1}$. The class of all domains $\mathcal{D} \subseteq \mathcal{R}(X)$ such that, for all $n$, the majority relation associated with any profile $\rho \in \mathcal{D}^{n}$ is acyclic has received significant attention in the literature, see the survey of Monjardet [2009] and the references therein. In the following, we will refer to any such domain as a Condorcet domain. $^{3}$

The following result prepares the ground for our analysis, providing some well-known characterizations of Condorcet domains (cf. [Monjardet, 2009, p. 142]). In particular, condition d) below is Sen's [1966] 'value restriction'; condition e) has been introduced by Ward [1965] as the 'absence of a Latin square' (in other terminology, it requires the absence of a 'Condorcet cycle'; cf. Condorcet [1785]).

Theorem 1. Let $X$ be finite, and let $\mathcal{D} \subseteq \mathcal{R}(X)$ be a subset of the space of all linear orders on $X$. The following statements are equivalent:

a) $\mathcal{D}$ is a Condorcet domain, i.e., the majority relation corresponding to every profile over $\mathcal{D}$ is acyclic.

b) For every profile over $\mathcal{D}$, the corresponding majority relation is a strict partial order (i.e., transitive and asymmetric binary relation).

\footnotetext{
${ }^{3}$ Fishburn [1997] calls them acyclic sets of linear orders.
} 
c) For every odd profile over $\mathcal{D}$, the corresponding majority relation is a linear order, i.e., an element of $\mathcal{R}(X)$.

d) For every triple $x, y, z \in X$ of pairwise distinct alternatives, there exists one element in $\{x, y, z\}$ that is either never ranked first, or never ranked second, or never ranked third in all restrictions of the orders in $\mathcal{D}$ to the set $\{x, y, z\}$.

e) For no triple $R_{1}, R_{2}, R_{3} \in \mathcal{D}$, and no triple $x, y, z \in X$ of pairwise distinct alternatives one has $x R_{1} y R_{1} z, y R_{2} z R_{2} x$ and $z R_{3} x R_{3} y$ simultaneously.

We will say that a Condorcet domain $\mathcal{D}$ is closed if the majority relation corresponding to any odd profile over $\mathcal{D}$ is again an element of $\mathcal{D}$, and we will say that a Condorcet domain $\mathcal{D}$ is maximal if no Condorcet domain (over the same set of alternatives) is a proper superset of $\mathcal{D}$. The following simple observation will be very useful.

Lemma 2.1. Let $\mathcal{D}$ be a Condorcet domain and $R \in \mathcal{R}(X)$ be the majority relation corresponding to an odd profile over $\mathcal{D}$. Then $\mathcal{D} \cup\{R\}$ is again a Condorcet domain. In particular, every Condorcet domain is contained in a closed Condorcet domain.

Proof. By Theorem 1e), it suffices to show that $\mathcal{D} \cup\{R\}$ does not admit three orders $R_{1}, R_{2}, R_{3}$ and three elements $x, y, z \in X$ such that $x R_{1} y R_{1} z, y R_{2} z R_{2} x$ and $z R_{3} x R_{3} y$. Assume on the contrary that it does; then, evidently, not all three orders $R_{1}, R_{2}, R_{3}$ belong to $\mathcal{D}$. Thus, one of them, say $R_{3}$, is the majority relation of an odd profile $\rho \in \mathcal{D}^{n}$, i.e. $R_{3}=R$. Consider the profile $\rho^{\prime}=\left(n R_{1}, n R_{2}, \rho\right) \in \mathcal{D}^{3 n}$ that consists of $n$ voters having the order $R_{1}, n$ voters having the order $R_{2}$ and the $n$ voters of the profile $\rho$. Then the voters of the subprofile $\left(n R_{1}, n R_{2}\right)$ will unanimously prefer $y$ to $z$, which forces the majority relation $P_{\rho^{\prime}}^{\text {maj }}$ corresponding to $\rho^{\prime}$ to have the same ranking of $y$ and $z$. At the same time, the voters of this subprofile are evenly split in the ranking of any other pair of alternatives from $\{x, y, z\}$. Hence, the majority relation $P_{\rho^{\prime}}^{\text {maj }}$ yields the cycle $z P_{\rho^{\prime}}^{\mathrm{maj}} x P_{\rho^{\prime}}^{\mathrm{maj}} y P_{\rho^{\prime}}^{\mathrm{maj}} z$, in contradiction to the assumption that $\mathcal{D}$ is a Condorcet domain.

This observation allows us to concentrate our attention on closed Condorcet domains without loss of generality, and we do so for the rest of the paper. Note, in particular, that by Lemma 2.1 all maximal Condorcet domains are closed.

\subsection{Betweenness and Median Domains}

The universal domain $\mathcal{R}(X)$ can be endowed with the ternary relation of 'Kemeny betweenness' [Kemeny, 1959]. According to it, an order $Q$ is between orders $R$ and $R^{\prime}$ if $Q \supseteq R \cap R^{\prime}$, i.e., $Q$ agrees with all binary comparisons on which $R$ and $R^{\prime}$ agree. ${ }^{4}$ The set of all orders that are between $R$ and $R^{\prime}$ is called the interval spanned by $R$ and $R^{\prime}$ and is denoted by $\left[R, R^{\prime}\right]$. This interval operator makes $\mathcal{R}(X)$ an interval space [van de Vel, $1993]^{5}$

\footnotetext{
${ }^{4}$ Some authors such as, e.g., Grandmont [1978] and Demange [2012] refer to orders that are between two others in this sense as 'intermediate' orders.

${ }^{5}$ For a closer analysis of this interval operator, see the appendix.
} 
A subset $\mathcal{D} \subseteq \mathcal{R}(X)$ is called median stable if, for any triple of elements $R_{1}, R_{2}, R_{3} \in \mathcal{D}$, there exists an element $R^{\text {med }}=R^{\text {med }}\left(R_{1}, R_{2}, R_{3}\right) \in \mathcal{D}$, the median order corresponding to $R_{1}, R_{2}, R_{3}$, such that

$$
R^{\text {med }} \in\left[R_{1}, R_{2}\right] \cap\left[R_{1}, R_{3}\right] \cap\left[R_{2}, R_{3}\right] .
$$

Proposition 2.1. The median order of a triple $R_{1}, R_{2}, R_{3} \in \mathcal{R}(X)$, if exists, is unique.

Proof. If a triple $R_{1}, R_{2}, R_{3}$ admits two different median orders, say $R$ and $R^{\prime}$, these must differ on the ranking of at least one pair of alternatives. Suppose they disagree on the ranking of $x$ and $y$. In this case, not all three orders of the triple agree on the ranking of $x$ versus $y$. Hence, exactly two of them, say $R_{1}$ and $R_{2}$, must agree on the ranking of $x$ versus $y$; but then, either $R$ or $R^{\prime}$ is not between $R_{1}$ and $R_{2}$, a contradiction.

In the following, we will refer to median stable subsets of $\mathcal{R}(X)$ as median domains. Evidently, not every subset of $\mathcal{R}(X)$ is a median domain; for instance, the universal domain $\mathcal{R}(X)$ itself is not a median domain whenever $|X| \geq 3$. This can be verified by considering any three orders of the form $R_{1}=\ldots a \ldots b \ldots c \ldots, R_{2}=\ldots b \ldots c \ldots a \ldots$, and $R_{3}=\ldots c \ldots a \ldots b \ldots$ Since any linear order $R$ in $\left[R_{1}, R_{3}\right]$ has $a R b$, any linear order $R$ in $\left[R_{1}, R_{2}\right]$ has $b R c$, and any linear order $R$ in $\left[R_{2}, R_{3}\right]$ has $c R a$, we obtain $\left[R_{1}, R_{2}\right] \cap\left[R_{1}, R_{3}\right] \cap\left[R_{2}, R_{3}\right]=\emptyset$ due to the transitivity requirement. Prominent examples of median domains include the well-studied single-crossing domains.

Example 1 (Classical single-crossing domains). There are several equivalent descriptions of single-crossing domains (see, e.g., Gans and Smart [1996], Saporiti [2009]). The following will be useful for our purpose. A domain $\mathcal{D} \subseteq \mathcal{R}(X)$ is said to have the single-crossing property if $\mathcal{D}$ can be linearly ordered, say according to $R_{1}>R_{2}>\ldots>$ $R_{m}$, so that, for all pairs $x, y$ of distinct elements of $X$, the sets $\left\{R_{j} \in \mathcal{D} \mid x R_{j} y\right\}$ and $\left\{R_{j} \in \mathcal{D} \mid y R_{j} x\right\}$ are connected in the order $>$. Thus, for each pair $x, y$ of distinct elements, there is exactly one 'cut-off' order $R_{k}$ such that either (i) $x R_{j} y$ for all $j \leq k$ and $y R_{j} x$ for all $j>k$, or (ii) $y R_{j} x$ for all $j \leq k$ and $x R_{j} y$ for all $j>k$. It is easily verified that, for any triple with $R_{i}>R_{j}>R_{k}$ the median order exists and coincides with the 'middle' order, i.e., $R^{\text {med }}\left(R_{i}, R_{j}, R_{k}\right)=R_{j}$.

The close connection between Condorcet domains and median domains to be established in Theorem 2 below stems from the following simple but fundamental observation (cf. [Nehring and Puppe, 2007, Cor. 5]).

Observation. A triple $R_{1}, R_{2}, R_{3} \in \mathcal{R}(X)$ admits a median order if and only if the majority relation of the profile $\rho=\left(R_{1}, R_{2}, R_{3}\right)$ is acyclic, in which case the median order $R^{\text {med }}\left(R_{1}, R_{2}, R_{3}\right)$ and the majority relation of $\rho$ coincide.

Proof. If the majority relation $P_{\rho}^{\text {maj }}$ is acyclic, and hence is an element of $\mathcal{R}(X)$, it belongs to each interval $\left[R_{i}, R_{j}\right]$ for all distinct $i, j \in\{1,2,3\}$. Indeed, if both $R_{i}$ and $R_{j}$ rank $x$ higher than $y$, then so does the majority relation. Conversely, if $R$ is the median of the triple $R_{1}, R_{2}, R_{3}$, then for any pair $x, y \in X$, at least two orders from this triple agree on ranking of $x$ and $y$. Then $R$ must agree with them, hence it is the majority relation for the profile $\rho=\left(R_{1}, R_{2}, R_{3}\right)$. 
Corollary 2.1. Any closed Condorcet domain is a median domain.

Proof. Suppose $\mathcal{D}$ is a closed Condorcet domain, and let $R_{1}, R_{2}, R_{3}$ be any triple of orders from $\mathcal{D}$. The majority relation $R$ corresponding to the profile $\left(R_{1}, R_{2}, R_{3}\right) \in \mathcal{D}^{3}$ by Theorem 1c) is an element of $\mathcal{R}(X)$, and by the assumed closedness it is in fact an element of $\mathcal{D}$. By the preceding observation, $R$ is the median order of the triple $R_{1}, R_{2}, R_{3}$.

A subset $\mathcal{C} \subseteq \mathcal{D}$ of a domain $\mathcal{D} \subseteq \mathcal{R}(X)$ will be called convex if $\mathcal{C}$ contains with any pair $R, R^{\prime} \in \mathcal{C}$ the entire interval spanned by $R$ and $R^{\prime}$, that is, $\mathcal{C}$ is convex if

$$
\left\{R, R^{\prime}\right\} \subseteq \mathcal{C} \Rightarrow\left[R, R^{\prime}\right] \subseteq \mathcal{C}
$$

A family $\mathbb{F}$ of subsets of a set is said to have the Helly property if the sets in any subfamily $\mathbb{F}^{\prime} \subseteq \mathbb{F}$ have a non-empty intersection whenever their pairwise intersections are non-empty, i.e., if $\mathcal{C} \cap \mathcal{C}^{\prime} \neq \emptyset$ for each pair $\mathcal{C}, \mathcal{C}^{\prime} \in \mathbb{F}^{\prime}$ implies $\cap \mathbb{F}^{\prime} \neq \emptyset$. For us this property will be important when $\mathbb{F}$ is the set of all convex subsets of a domain $\mathcal{D} \subseteq \mathcal{R}(X)$.

Proposition 2.2 (Helly property and median domains). A domain $\mathcal{D}$ is a median domain if and only if $\mathcal{D}$ has the Helly property for convex subsets.

Proof. Let $\mathcal{D}$ be median domain and $\mathbb{F}$ be a family of convex subsets with pairwise nonempty intersection. We proceed by induction over $m=|\mathbb{F}|$. If $m=2$, there is nothing to prove, thus let $m=3$, i.e., $\mathbb{F}=\left\{\mathcal{C}_{1}, \mathcal{C}_{2}, \mathcal{C}_{3}\right\}$. Choose any triple of orders $R_{1} \in \mathcal{C}_{1} \cap \mathcal{C}_{2}$, $R_{2} \in \mathcal{C}_{2} \cap \mathcal{C}_{3}$ and $R_{3} \in \mathcal{C}_{3} \cap \mathcal{C}_{1}$, and consider the median order $R=R^{\text {med }}\left(R_{1}, R_{2}, R_{3}\right)$. By convexity of the sets $\mathcal{C}_{1}, \mathcal{C}_{2}, \mathcal{C}_{3}$ we have $R \in \mathcal{C}_{1} \cap \mathcal{C}_{2} \cap \mathcal{C}_{3}$ which, in particular, shows that $\cap \mathbb{F}$ is non-empty. Now consider $\mathbb{F}=\left\{\mathcal{C}_{1}, \ldots, \mathcal{C}_{m}\right\}$ with $m>3$ elements, and assume that the assertion holds for all families with less than $m$ elements. Then, the family $\left\{\mathcal{C}_{1}, \mathcal{C}_{2}, \mathcal{C}_{3} \cap \ldots \cap \mathcal{C}_{m}\right\}$ constitutes a family of three convex subsets with pairwise nonempty intersections. By the preceding argument, we thus have $\cap \mathbb{F} \neq \emptyset$.

Conversely, consider a domain $\mathcal{D}$ such that any family of convex subsets of $\mathcal{D}$ has the Helly property. Consider any three orders $R_{1}, R_{2}, R_{3} \in \mathcal{D}$. Since, evidently, all intervals are convex, the Helly property applied to the intervals $\left[R_{1}, R_{2}\right],\left[R_{1}, R_{3}\right],\left[R_{2}, R_{3}\right]$ implies the existence of a median.

For any domain $\mathcal{D}$ and any pair $x, y \in X$ of alternatives, denote by $\mathcal{V}_{x y}^{\mathcal{D}}$ the set of orders in $\mathcal{D}$ that rank $x$ above $y$, i.e.,

$$
\mathcal{V}_{x y}^{\mathcal{D}}:=\{R \in \mathcal{D} \mid x R y\}
$$

Note that, for all distinct $x, y \in X$, the sets $\mathcal{V}_{x y}^{\mathcal{D}}$ and $\mathcal{V}_{y x}^{\mathcal{D}}$ form a partition of $\mathcal{D}$. Also observe that the sets of the form $\mathcal{V}_{x y}^{\mathcal{D}}$ are convex for all pairs $x, y \in X$. We will now use the Helly property applied to this family of convex sets to show that every median domain is a closed Condorcet domain. The following is the main result of this section.

Theorem 2. The classes of median domains and closed Condorcet domains coincide, i.e., a domain is a median domain if and only if it is a closed Condorcet domain. 
Proof. In the light of Corollary 2.1, it suffices to show that every median domain is a closed Condorcet domain. Thus, let $\mathcal{D}$ be a median domain and consider an odd profile $\rho=\left(R_{1}, \ldots, R_{n}\right) \in \mathcal{D}^{n}$. For any two alternatives $x, y \in X$, let $\mathcal{U}_{x y}=\left\{R_{i} \mid x R_{i} y\right\}$, and observe that obviously, $\mathcal{U}_{x y} \subseteq \mathcal{V}_{x y}^{\mathcal{D}}$. Let $z, w$ also be alternatives in $X$, not necessarily distinct from $x$ and $y$. If $x P_{\rho}^{\text {maj }} y$ and $z P_{\rho}^{\text {maj }} w$, then $\mathcal{U}_{x y} \cap \mathcal{U}_{z w} \neq \emptyset$ and hence $\mathcal{V}_{x y}^{\mathcal{D}} \cap \mathcal{V}_{z w}^{\mathcal{D}} \neq \emptyset$. By Proposition 2.2 we have

$$
\bigcap_{x, y \in X: x P_{\rho}^{\text {maj }} y} \mathcal{V}_{x y}^{\mathcal{D}} \neq \emptyset,
$$

hence there is a linear order in $\mathcal{D}$ which coincides with the majority relation of $\rho$.

\section{Closed Condorcet Domains and Median Graphs}

The close relation between the majority relation and the median operator in closed Condorcet domains suggests that there exists a deeper structural connection between closed Condorcet domains and median graphs. ${ }^{6}$ The details of this connection are worked out in this section. We start in Subsection 3.1 with some basic facts about median graphs. In Subsection 3.2 we prove that the graph associated with any closed Condorcet domain is a median graph. In Subsection 3.3 we show that, conversely, for every median graph one can construct a (non-unique) closed Condorcet domain whose associated graph is isomorphic to the given graph.

\subsection{Median Graphs}

Let $\Gamma=(V, E)$ be a connected graph. The distance $d(u, v)$ between two vertices $u, v \in V$ is the smallest number of edges that a path connecting $u$ and $v$ may contain. While the distance is uniquely defined, there may be several shortest paths from $u$ to $v$. We say that a vertex $w$ is geodesically between the vertices $u$ and $v$ if $w$ lies on a shortest path that connects $u$ and $v$ or, equivalently, if $d(u, v)=d(u, w)+d(w, v)$. A (geodesically) convex set in a graph $\Gamma=(V, E)$ is a subset $C \subseteq V$ such that for any two vertices $u, v \in C$ all vertices on every shortest path between $u$ and $v$ in $\Gamma$ lie in $C$. A connected graph $\Gamma=(V, E)$ is called a median graph if, for any three vertices $u, v, w \in V$, there is a unique vertex $\operatorname{med}(u, v, w) \in V$ which lies simultaneously on some shortest paths from $u$ to $v$, from $u$ to $w$ and from $v$ to $w$.

To characterize the structure of an arbitrary median graph we recall the concept of convex expansion. For any two subsets $S, T \subseteq V$ of the set of vertices of the graph $\Gamma$, let $E(S, T) \subseteq E$ denote the set of edges that connect vertices in $S$ and vertices in $T$.

Definition 1. Let $\Gamma=(V, E)$ be a graph. Let $W_{1}, W_{2} \subset V$ be two subsets with a nonempty intersection $W_{1} \cap W_{2} \neq \emptyset$ such that $W_{1} \cup W_{2}=V$ and $E\left(W_{1} \backslash W_{2}, W_{2} \backslash W_{1}\right)=\emptyset$. The expansion of $\Gamma$ with respect to $W_{1}$ and $W_{2}$ is the graph $\Gamma^{\prime}$ constructed as follows:

\footnotetext{
${ }^{6}$ The term 'median graph' was coined by Nebeský [1971]; for a comprehensive survey on median graphs see Klavzar and Mulder [1999].
} 

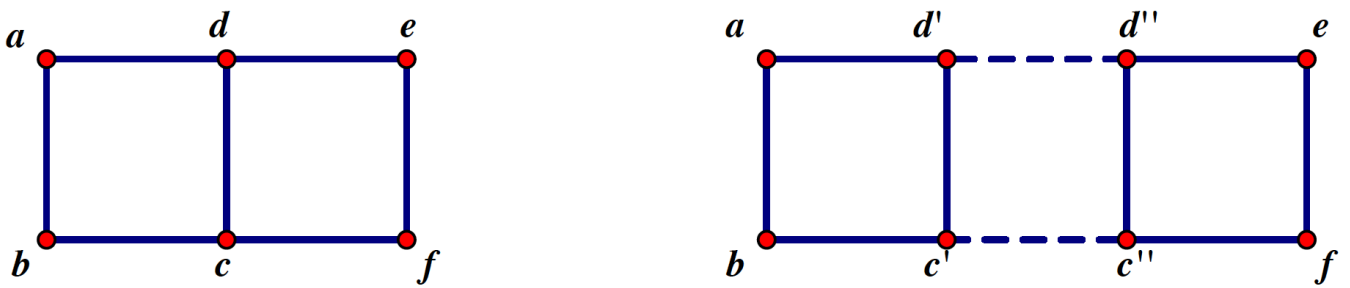

Figure 1: Convex expansion of a median graph

- each vertex $v \in W_{1} \cap W_{2}$ is replaced by two vertices $v^{1}, v^{2}$ joined by an edge;

- $v^{1}$ is joined to all the neighbors of $v$ in $W_{1} \backslash W_{2}$ and $v^{2}$ is joined to all the neighbors of $v$ in $W_{2} \backslash W_{1}$;

- if $v, w \in W_{1} \cap W_{2}$ and $v w \in E$, then $v^{1}$ is joined to $w^{1}$ and $v^{2}$ is joined to $w^{2}$;

- if $v, w \in W_{1} \backslash W_{2}$ or if $v, w \in W_{2} \backslash W_{1}$, they will be joined by an edge in $\Gamma^{\prime}$ if and only if they were joined in $\Gamma$; if $v \in W_{1} \backslash W_{2}$ and $w \in W_{2} \backslash W_{1}$, they remain not joined in $\Gamma^{\prime}$.

If $W_{1}$ and $W_{2}$ are convex, then $\Gamma^{\prime}$ will be called a convex expansion of $\Gamma$.

Example 2 (Convex expansion). In the graph $\Gamma$ shown on the left of Figure 1 we set $W_{1}=\{a, b, c, d\}$ and $W_{2}=\{c, d, e, f\}$. These are convex and their intersection $W_{1} \cap W_{2}=\{c, d\}$ is not empty. On the right we see the graph $\Gamma^{\prime}$ obtained by the convex expansion of $\Gamma$ with respect to $W_{1}$ and $W_{2}$.

The following important theorem about median graphs is due to Mulder [1978].

Theorem 3 (Mulder's convex expansion theorem). A graph is median if and only if it can be obtained from a trivial one-vertex graph by repeated convex expansions.

\subsection{Every Closed Condorcet Domain Induces a Median Graph}

As we already said in the introduction, with every domain $\mathcal{D} \subseteq \mathcal{R}(X)$ one can associate a graph $\Gamma_{\mathcal{D}}$ on $\mathcal{D}$ as follows. Two distinct orders $R, R^{\prime} \in \mathcal{D}$ are said to be neighbors in $\mathcal{D}$, or simply $\mathcal{D}$-neighbors, if $\left[R, R^{\prime}\right] \cap \mathcal{D}=\left\{R, R^{\prime}\right\}$. Define $\Gamma_{\mathcal{D}}$ to be the (undirected) graph on $\mathcal{D}$ that connects each pair of $\mathcal{D}$-neighbors by an edge. We say that $\Gamma_{\mathcal{D}}$ is the associated graph of $\mathcal{D}$. We recall that the graph associated with the universal domain $\mathcal{R}(X)$ is called the permutohedron. Note that the graph $\Gamma_{\mathcal{D}}$ is always connected, i.e., any two orders in $\mathcal{D}$ are connected by a path in $\Gamma_{\mathcal{D}}$. Moreover, any two $\mathcal{R}(X)$-neighbors $R, R^{\prime}$ are always $\mathcal{D}$ neighbors whenever $R, R^{\prime} \in \mathcal{D}$. However, two $\mathcal{D}$-neighbors need not be $\mathcal{R}(X)$-neighbors, so, if $\mathcal{D} \neq \mathcal{R}(X)$, the associated graph $\Gamma_{\mathcal{D}}$ need not be a subgraph of the permutohedron. If it is, the domain $\mathcal{D}$ is called connected. ${ }^{7}$

\footnotetext{
${ }^{7}$ Since connectedness of a domain is thus not the same as connectedness of the associated graph, the terminology might be a bit confusing. But it is widely accepted in the literature [Monjardet, 2009].
} 
We will now define another concept of betweenness, different from the Kemeny one. For any $\mathcal{D} \subseteq \mathcal{R}(X)$, the order $Q \in \mathcal{D}$ is $\Gamma_{\mathcal{D}}$-geodesically between the orders $R, R^{\prime} \in \mathcal{D}$ if $Q$ lies on a shortest $\Gamma_{\mathcal{D}}$-path that connects $R$ and $R^{\prime}$.

As a first example, consider Figure 2 which depicts two single-crossing domains on the set $X=\{a, b, c\}$ with their corresponding graphs. The domain on the left is $\mathcal{D}_{1}=$ $\{a b c, a c b, c a b, c b a\}$, the domain on the right is $\mathcal{D}_{2}=\{a b c, b a c, b c a, c b a\}$.
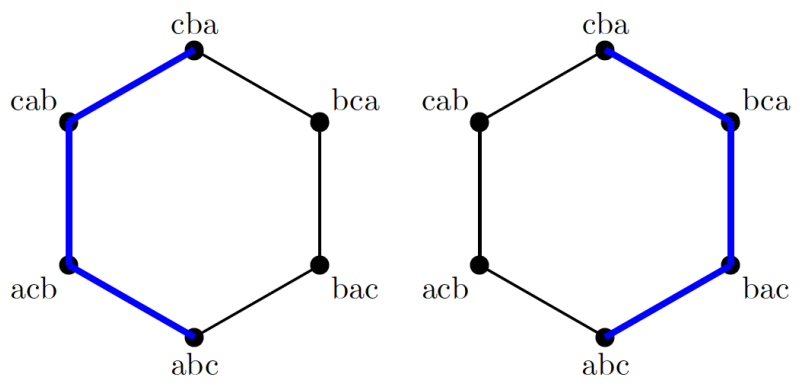

Figure 2: Two single-crossing domains on $\{a, b, c\}$ with their associated graphs

Note that the permutohedron is given by the entire 6-cycle, and that the graphs associated with the two single-crossing domains are linear subgraphs of the permutohedron. In particular, both domains are connected and the Kemeny betweenness relation on $\mathcal{D}_{1}$ and $\mathcal{D}_{2}$ translates into the geodesic betweenness of their associated graphs.

It is important to note that for an arbitrary domain $\mathcal{D}$ both of these properties may not be true: neither is $\Gamma_{\mathcal{D}}$ in general a subgraph of $\Gamma_{\mathcal{R}(X)}$, nor do the Kemeny betweenness on $\mathcal{D}$ and the geodesic betweenness on $\Gamma_{\mathcal{D}}$ correspond to each other. To illustrate this, consider the three domains with their associated graphs depicted in Figure 3. Evidently, none of the three graphs is a subgraph of the permutohedron, hence none of the corresponding domains is connected. As is easily verified, the domain $\{a b c, a c b, c b a, b c a\}$ on the left of Figure 3 is a median domain, but the two other domains are not; for instance, the domain $\{a b c, c a b, c b a, b c a\}$ in the middle of Figure 3 contains a cyclic triple of orders $a b c, b c a$ and $c a b$, and the 5-element domain $\{a c b, c a b, c b a, b c a, b a c\}$ on the right of Figure 3 contains the cyclic triple $a c b, c b a$ and $b a c$.
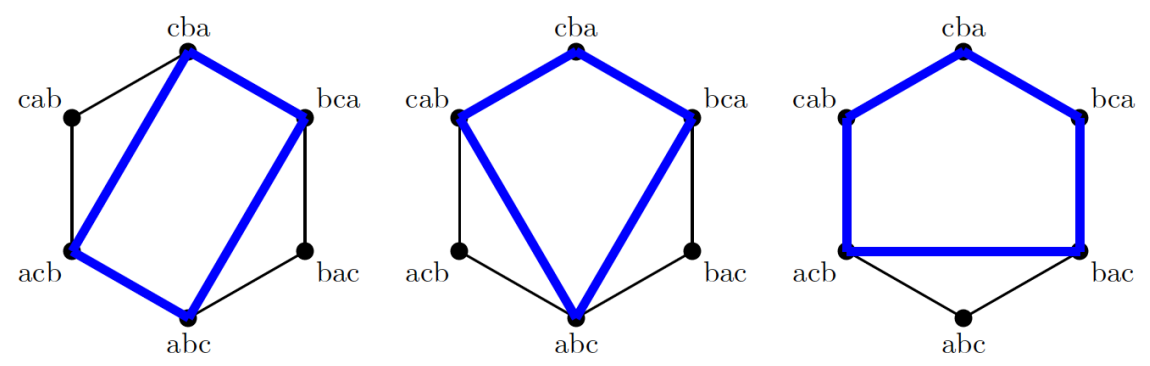

Figure 3: Three domains on $\{a, b, c\}$ with their associated graphs 
In case of the domain on the left-hand side, the Kemeny betweenness on $\mathcal{D}$ and the geodesic betweenness on the associated graph $\Gamma_{\mathcal{D}}$ agree. By contrast, for the domain in the middle we have $a b c \notin[c a b, b c a]$ but evidently $a b c$ is geodesically between the orders $c a b$ and $b c a$ in the associated graph. On the other hand, in this example the Kemeny betweenness implies geodesic betweenness. But this also does not hold in general, as the domain on the right of Figure 3 shows: here, both orders $c a b$ and $c b a$ are elements of $[a c b, b c a]$, but neither of them is geodesically between $a c b$ and $b c a$.

For any domain $\mathcal{D} \subseteq \mathcal{R}(X)$, we now have two betweenness relations on $\mathcal{D}$ : the Kemeny betweenness and the geodesic betweenness in the associated graph $\Gamma_{\mathcal{D}}$. We will show that in important cases they coincide, in which case for all $R, R^{\prime}, Q \in \mathcal{D}$, we have

$$
Q \in\left[R, R^{\prime}\right] \Leftrightarrow Q \text { is } \Gamma_{\mathcal{D}} \text {-geodesically between } R \text { and } R^{\prime} .
$$

The following lemma is central to our approach. It states that the Kemeny betweenness on a domain coincides with the geodesic betweenness of its associated graph for three natural classes of domains: (i) median domains, (ii) connected domains, and (iii) domains for which the associated graph is acyclic.

Lemma 3.1. The Kemeny betweenness on a domain $\mathcal{D} \subseteq \mathcal{R}(X)$ coincides with the geodesic betweenness of the associated graph $\Gamma_{\mathcal{D}}$, if one of the following conditions is satisfied:

(i) $\mathcal{D}$ is a median domain,

(ii) $\mathcal{D}$ is connected,

(iii) $\Gamma_{\mathcal{D}}$ is acyclic (i.e., a tree).

The proof of this lemma is provided in the appendix and invokes a general result on 'geometric interval operators' satisfying the so-called triangle condition due to Bandelt and Chepoi [1996].

From Theorem 2 and Lemma 3.1(i), we immediately obtain the central result of this section.

Theorem 4. The associated graph $\Gamma_{\mathcal{D}}$ of any closed Condorcet domain $\mathcal{D}$ is a median graph. Moreover, the Kemeny betweenness relation on $\mathcal{D}$ coincides with the geodesic betweenness on $\Gamma_{\mathcal{D}}$.

We again stress that not all median domains are connected, as exemplified by the domain on the left-hand-side of Figure 3. It is also worth noting that Lemma 3.1 does not imply that $\mathcal{D}$ is a median domain whenever the associated graph $\Gamma_{\mathcal{D}}$ is median graph. A counterexample is the domain $\mathcal{D}$ in the middle of Figure 3, which is not a median domain despite the fact that its graph $\Gamma_{\mathcal{D}}$ is a median graph. However, we have the following corollary.

Corollary 3.1. Let $\mathcal{D} \subseteq \mathcal{R}(X)$ be a connected domain. Then, $\mathcal{D}$ is a median domain if and only if the associated graph $\Gamma_{\mathcal{D}}$ is a median graph. 
Proof. The associated graph of any median domain is a median graph by Lemma 3.1(i). Conversely, if $\mathcal{D}$ is connected the geodesic median of any triple of vertices with respect to $\Gamma_{\mathcal{D}}$ is also the median with respect to the Kemeny betweenness in $\mathcal{D}$ by Lemma 3.1(ii). Thus, if $\Gamma_{\mathcal{D}}$ is a median graph, $\mathcal{D}$ is a median domain.

As another important corollary of our analysis, we obtain that all closed Condorcet domains that contain two completely reversed orders have the structure of a distributive lattice. Say that two orders $\bar{R}, \underline{R} \in \mathcal{R}(X)$ are completely reversed if $\bar{R} \cap \underline{R}=\{(x, x) \mid x \in$ $X$ \}, i.e., if $\bar{R}$ and $\underline{R}$ agree on the ranking of no pair of distinct alternatives.

Corollary 3.2. Let $\mathcal{D}$ be a closed Condorcet domain. If $\mathcal{D}$ contains at least two completely reversed orders, then it is a distributive lattice.

Proof. By Theorem 4, the graph $\Gamma_{\mathcal{D}}$ associated with $\mathcal{D}$ is a median graph. Let $\bar{R}, \underline{R} \in \mathcal{D}$ be two completely reversed orders, and define the join and meet operations on $\mathcal{D}$ by $R \vee R^{\prime}:=R^{\operatorname{med}}\left(R, \bar{R}, R^{\prime}\right)$ and $R \wedge R^{\prime}:=R^{\operatorname{med}}\left(R, \underline{R}, R^{\prime}\right)$, respectively. It is easy (though tedious) to verify that with these operations the space $\mathcal{D}$ has the structure of a distributive lattice. Another way to prove the corollary is by invoking a well-known result by Avann [1961] which states that a median graph is (the covering graph of) a distributive lattice if and only if it contains two vertices, say 1 and 0 , such that every vertex is on a shortest path connecting 1 and 0 . If one takes 1 to be $\bar{R}$ and 0 to be $\underline{R}$, it is easily seen using Lemma 3.1(i) that $\Gamma_{\mathcal{D}}$ satisfies all premises of Avann's result.

Remark. Chameni-Nembua [1989] and Abello [1991] have shown that all maximal and connected Condorcet domains that contain at least two completely reversed orders have the structure of a distributive lattice. This result was generalized by Danilov and Koshevoy [2013] who showed that the connectedness is in fact not needed for the conclusion. Corollary 3.2 further generalizes this by showing that the condition of maximality can be substantially weakened to the condition of closedness. In fact, even without the closedness condition, one would still obtain that every Condorcet domain that contains two completely reversed orders can be embedded in a distributive lattice. On the other hand, the condition that the domain contain at least one pair of completely reversed orders cannot be dropped (an example illustrating this is given in Figure 8 below).

\subsection{Every Median Graph is Associated with a Closed Condorcet Domain}

Is every median graph induced by some closed Condorcet domain? The following result gives an affirmative answer. Interestingly, we will see later in Section 5, that the answer becomes negative if we insist on maximality of the Condorcet domain, i.e., there exist median graphs that cannot be associated with any maximal Condorcet domain.

Theorem 5. For every (finite) median graph $\Gamma=(V, E)$ there exists a closed Condorcet domain $\mathcal{D} \subseteq \mathcal{R}(Y)$ on a finite set of alternatives $Y$ with $|Y| \leq|V|$ such that $\Gamma_{\mathcal{D}}$ is isomorphic to $\Gamma$. 
Proof. We apply Mulder's theorem. Since the statement is true for the trivial graph consisting of a single vertex, arguing by induction, we assume that the statement is true for all median graphs with $k$ vertices or less. Let $\Gamma^{\prime}=\left(V^{\prime}, E^{\prime}\right)$ be a median graph with $\left|V^{\prime}\right|=k+1$. By Mulder's theorem $\Gamma^{\prime}$ is a convex expansion of some median graph $\Gamma=(V, E)$ relative to convex subsets $W_{1}$ and $W_{2}$, where $|V|=\ell \leq k$. By induction there exists a domain $\mathcal{D} \subseteq \mathcal{R}(X)$ with $|X| \leq k$ such that $\Gamma_{\mathcal{D}}$ is isomorphic to $\Gamma$ with the mapping $R: v \mapsto R_{v}$ associating a linear order $R_{v} \in \mathcal{D}$ to a vertex $v \in V$.

To obtain a new domain $\mathcal{D}^{\prime}$ such that $\Gamma_{\mathcal{D}^{\prime}}$ is isomorphic to $\Gamma^{\prime}$ we clone an arbitrary alternative $x \in X$ and introduce a clone ${ }^{8} y \notin X$ of $x$ and denote $X^{\prime}=X \cup\{y\}$. The mapping $R^{\prime}: v \mapsto R_{v}^{\prime}$ that associates any vertex $v \in \Gamma^{\prime}$ to a linear order $R_{v}^{\prime}$ over $X^{\prime}$ will be constructed as follows. If $v$ is a vertex of $W_{1} \backslash W_{2}$, to obtain $R_{v}^{\prime}$ we replace $x$ with $x y$ in $R_{v}$, placing $x$ higher than $y$, and to obtain $R_{u}^{\prime}$ for $u \in W_{2} \backslash W_{1}$ we replace $x$ by $y x$ in $R_{u}$, placing $y$ higher than $x$. Let $v$ now be in $W_{1} \cap W_{2}$. In the convex expansion this vertex is split into $v^{1}$ and $v^{2}$. To obtain $R_{v^{1}}^{\prime}$ we clone the linear order $R_{v}$ replacing $x$ by $x y$ and to obtain $R_{v^{2}}^{\prime}$ we clone the same linear order $R_{v}$ replacing $x$ by $y x$. The number of alternatives has increased by one only, so it is not greater than $\left|V^{\prime}\right|=\ell+1 \leq k+1$.

To prove that $\Gamma_{\mathcal{D}^{\prime}}$ is isomorphic to $\Gamma^{\prime}$ we must prove that $R_{u}^{\prime}$ and $R_{v}^{\prime}$ are neighbors if and only if $u, v \in V^{\prime}$ are.

First, we need to show that there is no edge between $R_{u}^{\prime}$ and $R_{v}^{\prime}$ if $v \in W_{1} \backslash W_{2}$ and $u \in W_{2} \backslash W_{1}$ since $u$ and $v$ are not neighbors in $\Gamma^{\prime}$. This follows from the fact that $u$ and $v$ were not neighbors in $\Gamma^{\prime}$ and hence $R_{u}$ and $R_{v}$ were not neighbors in $\Gamma_{\mathcal{D}}$. Hence there was a linear order $R_{w} \in\left[R_{u}, R_{v}\right]$ between them. In $\mathcal{D}^{\prime}$ this linear order will be cloned to $R_{w}^{\prime}$ and, no matter how we place $x$ and $y$ there, we obtain $R_{w}^{\prime} \in\left[R_{u}^{\prime}, R_{v}^{\prime}\right]$ since $R_{u}^{\prime}$ and $R_{v}^{\prime}$ disagree on $x$ and $y$. Hence $R_{u}^{\prime}$ and $R_{v}^{\prime}$ are not neighbors in $\Gamma_{\mathcal{D}^{\prime}}$ as well. Secondly, we have to check that $R_{v^{1}}^{\prime}$ and $R_{v^{2}}^{\prime}$ are linked by an edge since $v^{1}$ and $v^{2}$ are neighbors in $\Gamma^{\prime}$. This holds because these orders differ in the ranking of just one pair of alternatives, namely $x$ and $y$, hence they are neighbors in $\Gamma_{\mathcal{D}^{\prime}}$. If $w \in W_{1} \cap W_{2}$ was a neighbor of $v \in W_{1} \backslash W_{2}$ in $\Gamma$, then $w^{1}$ is a neighbor of $v$ in $\Gamma^{\prime}$ but $w^{2}$ is not. We then have that $R_{w}$ is a neighbor of $R_{v}$ in $\Gamma_{\mathcal{D}}$. Then $R_{w^{1}}^{\prime}$ will obviously be a neighbor of $R_{v}^{\prime}$ in $\Gamma_{\mathcal{D}^{\prime}}$ while $R_{w^{2}}^{\prime}$ will not be a neighbor of $R_{v}^{\prime}$ in $\Gamma_{\mathcal{D}^{\prime}}$ since $R_{w^{1}}^{\prime}$ will be between them. The remaining cases are considered similarly.

To complete the induction step we need to prove that $\mathcal{D}^{\prime}$ is a Condorcet domain. We use Theorem 1d) for that. We need to consider only triples of elements of $X^{\prime}$ that contain $x$ and $y$. Let $\{a, x, y\}$ be such a triple. Since in orders of $\mathcal{D}^{\prime}$ elements $x$ and $y$ are always standing together, $a$ can never be in the middle. Hence conditions of Theorem 1d) are satisfied and $\mathcal{D}^{\prime}$ is a Condorcet domain.

Clearwater et al. [2015] showed that for a median graph with $k$ vertices we might need exactly $k$ alternatives to construct a closed Condorcet domain that has the given graph as associated graph, with the star-graph representing the worst-case scenario. Thus, Theorem 5 cannot be improved in this respect.

\footnotetext{
${ }^{8}$ We say that $x$ and $y$ are clones if they are neighbors in any linear order in the domain, cf. Elkind et al. [2011, 2012].
} 
Relation to Demange [2012]. We conclude this section by commenting on the relation to Demange [2012]. She introduces profiles of preferences that are parametrized by a median graph and derives the acyclicity of the majority relation from the requirement of intermediateness (cf. Grandmont [1978]) of preferences. Specifically, she considers a median graph with set of vertices $V$ (identified with the set of voters), and a collection $\left\{R_{v} \in \mathcal{R}(X): v \in V\right\}$ of linear orders and assumes that, for all $v, v^{\prime}, u \in V$, the following condition is satisfied:

$$
R_{u} \in\left[R_{v}, R_{v^{\prime}}\right] \text { whenever } u \text { is on a shortest path between } v \text { and } v^{\prime} .
$$

Lemma 3.1(i) above shows that, for every median domain $\mathcal{D}$, the intermediateness condition (3.2) is always satisfied with respect to the geodesic betweenness of the underlying graph $\Gamma_{\mathcal{D}}$. Thus, Theorem 4 above implies that in fact all closed Condorcet domains are of the kind considered by Demange, and demonstrates the existence of a 'canonical parametrization' of these domains (namely by the induced graph $\Gamma_{\mathcal{D}}$ ). Finally, Demange [2012] takes both the (median) graph and the corresponding profile as given. We show that this does not impose any restriction on the graph since, by Theorem 5 , for every median graph there exists a domain satisfying the intermediateness requirement (3.2). ${ }^{9}$

\section{Characterizing and Generalizing the Single-Crossing Property}

\subsection{The Representative Voter Property}

A representative voter for a given profile of linear orders is a voter, present in this profile, whose preference coincides with the majority relation of the profile. We say that a domain $\mathcal{D}$ has the representative voter property if in any odd profile composed of linear orders from $\mathcal{D}$ admits a representative voter.

In this subsection, we prove that the single-crossing domains (cf. Example 1 above) are exactly the median domains whose associated graphs are chains. From this, we obtain that - with the exception of the Condorcet domains associated with the 4-cycle graph the single-crossing domains are the domains that have the representative voter property.

Proposition 4.1. A domain $\mathcal{D} \subseteq \mathcal{R}(X)$ has the single-crossing property if and only if its associated graph $\Gamma_{\mathcal{D}}$ is a chain.

Proof. It is easily seen that, if $\mathcal{D}=\left\{R_{1}, \ldots, R_{m}\right\}$ is single-crossing with respect to the linear order $R_{1}>R_{2}>\ldots>R_{m}$, then the interval $\left[R_{i}, R_{j}\right]$ for $i<j$ consists of linear orders $R_{i}, R_{i+1}, \ldots, R_{j}$. Hence, the graph $\Gamma_{\mathcal{D}}$ is given by the chain connecting $R_{j}$ and $R_{j+1}$ by an edge for all $j=1, \ldots, m-1$.

Conversely, suppose that $\mathcal{D}=\left\{R_{1}, \ldots, R_{m}\right\}$ is such that the associated graph $\Gamma_{\mathcal{D}}$ is a chain, say the one that connects $R_{j}$ with $R_{j+1}$ by an edge for all $j=1, \ldots, m-1$. We show that $\mathcal{D}$ is single-crossing with respect to the linear order $R_{1}>R_{2}>\ldots>R_{m}$. By

\footnotetext{
${ }^{9}$ In contrast to the present analysis, Demange [2012] also considers the generalization to weak orders.
} 
Lemma 3.1(iii), the geodesic betweenness in $\Gamma_{\mathcal{D}}$ agrees with the Kemeny betweenness of $\mathcal{D}$ as a subset of $\mathcal{R}(X)$, hence, if $R_{h}, R_{l} \in \mathcal{D}$ with $l>h$ are such that $x R_{h} y$ and $x R_{l} y$, then we also have $x R_{j} y$ for all $j \in\{h, \ldots, l\}$ and all $x, y \in X$. This implies that $\mathcal{D}$ has the single-crossing property relative to the specified order.

Rothstein [1991] proved the sufficiency of the single-crossing property for the validity of the representative voter property. The following result shows that the single-crossing property is in fact 'almost' necessary.

Theorem 6. Let $\mathcal{D} \subseteq \mathcal{R}(X)$ be a domain of linear orders on $X$. Then, $\mathcal{D}$ has the representative voter property if and only if $\mathcal{D}$ is either a single-crossing domain, or $\mathcal{D}$ is a closed Condorcet domain with exactly four elements such that the associated graph $\Gamma_{\mathcal{D}}$ is a 4-cycle.

Proof. Suppose that $\mathcal{D}$ has the representative voter property. Evidently, in this case $\mathcal{D}$ is a closed Condorcet domain. By Theorem 4, the associated graph $\Gamma_{\mathcal{D}}$ is median, and by Lemma 3.1(i) the geodesic betweenness in $\Gamma_{\mathcal{D}}$ agrees with the Kemeny betweenness on $\mathcal{D}$. We will show that all vertices of $\Gamma_{\mathcal{D}}$ have degree at most 2 . Suppose by way of contradiction, that $\Gamma_{\mathcal{D}}$ contains a vertex, say $R$, of degree at least 3 . Consider any profile $\rho=\left(R_{1}, R_{2}, R_{3}\right)$ consisting of three distinct neighbors of $R$. The majority relation corresponding to $\rho$ is the median $R^{\text {med }}\left(R_{1}, R_{2}, R_{3}\right)$. Since the median graph $\Gamma_{\mathcal{D}}$ does not have 3-cycles, $R_{i}$ and $R_{j}$ cannot be neighbors for all distinct $i, j \in\{1,2,3\}$, hence $R^{\text {med }}\left(R_{1}, R_{2}, R_{3}\right)=R$. Since $R$ is not an element of $\left\{R_{1}, R_{2}, R_{3}\right\}$ the representative voter property is violated, a contradiction. Since $\Gamma_{\mathcal{D}}$ is always connected (as a graph), the absence of vertices of degree 3 or more implies that $\Gamma_{\mathcal{D}}$ is either a cycle or a chain. It is well-known that among all cycles, only 4-cycles are a median graphs. ${ }^{10}$ On the other hand, if $\Gamma_{\mathcal{D}}$ is a chain, then $\mathcal{D}$ has the single-crossing property by Proposition 4.1.

To prove the converse, suppose first that $\mathcal{D}$ is a single-crossing domain. Then $\Gamma_{\mathcal{D}}$ is a chain by Proposition 4.1 and, evidently, the preference of the median voter in any odd profile coincides with the corresponding majority relation; this is Rothstein's theorem (Rothstein [1991]). On the other hand, consider any odd profile over a domain $\mathcal{D}$ such that the induced graph is a 4-cycle. In that case, the representative voter property holds trivially if the profile contains all four different orders; if it contains at most three different orders, the representative voter property follows as in the case of a chain with at most three elements.

\subsection{Generalizing the Single-Crossing Property to Trees}

The classical single-crossing property [Mirrlees, 1971, Gans and Smart, 1996] naturally generalizes to trees. We rephrase the definition suggested by Kung [2014] and Clearwater et al. [2015] as follows.

\footnotetext{
${ }^{10}$ Clearly, a 3 -cycle is not a median graph. Moreover, for any $k \geq 5$, one can find three vertices $v_{1}, v_{2}, v_{3}$ on a $k$-cycle such that the three shortest paths between any pair from $v_{1}, v_{2}, v_{3}$ cover the entire cycle; this implies that no vertex can simultaneously lie on all three shortest paths, i.e., that the triple $v_{1}, v_{2}, v_{3}$ does not admit a median.
} 
Definition 2. A domain $\mathcal{D} \subseteq \mathcal{R}(X)$ is single-crossing with respect to the tree $T=(V, E)$ if $|\mathcal{D}|=|V|$ and the linear orders of $\mathcal{D}$ can be parametrized by the vertices of $T$ so that the intermediateness condition (3.2) is satisfied. Moreover, we say that a domain is generalized single-crossing if it is single-crossing with respect to some tree.

As is easily verified, a domain $\mathcal{D}$ is generalized single-crossing if and only if its associated graph $\Gamma_{\mathcal{D}}$ is a tree. Indeed, if a domain $\mathcal{D}$ is single-crossing with respect to the tree $T$, then $\Gamma_{\mathcal{D}}=T$. Conversely, if $\Gamma_{\mathcal{D}}$ is a tree, then by Lemma 3.1(iii), the preferences of $\mathcal{D}$ are intermediate on $\Gamma_{\mathcal{D}}$ so $\mathcal{D}$ is generalized single-crossing. In particular, every generalized single-crossing domain is a closed Condorcet domain by Lemma 3.1(iii) (cf. Demange [2012], Clearwater et al. [2015]).

To justify our terminology of 'generalized single-crossingness,' let $\mathcal{D} \subseteq \mathcal{R}(X)$ be a generalized single-crossing domain and suppose not all orders in $\mathcal{D}$ agree on the ranking of $x, y \in X$, then the sets $\mathcal{V}_{x y}^{\mathcal{D}}:=\{R \in \mathcal{D} \mid x R y\}$ and $\mathcal{V}_{y x}^{\mathcal{D}}:=\{R \in \mathcal{D} \mid y R x\}$ are both nonempty. In the tree $\Gamma_{\mathcal{D}}$, there will be a unique edge $Q R$ such that $Q \in \mathcal{V}_{x y}^{\mathcal{D}}$ and $R \in \mathcal{V}_{y x}^{\mathcal{D}}$; moreover, both sets $\mathcal{V}_{x y}^{\mathcal{D}}$ and $\mathcal{V}_{y x}^{\mathcal{D}}$ are convex, and $\mathcal{V}_{x y}^{\mathcal{D}} \cup \mathcal{V}_{y x}^{\mathcal{D}}=\mathcal{D}$.

Example 3 (The single-crossing property on a tree). Consider domain $\mathcal{D}$ on the set $\{a, b, c, d\}$ consisting of four orders: $\hat{R}=a b c d, R_{1}=a c b d, R_{2}=a b d c$, and $R_{3}=b a c d$. As is easily seen, $\mathcal{D}$ is a closed Condorcet domain. The associated graph $\Gamma_{\mathcal{D}}$ connects $\hat{R}$ with each of the other three orders by an edge and the graph has no other edges (cf. Figure 4). Thus, $\Gamma_{\mathcal{D}}$ is a tree and $\hat{R}$ is the median order of any triple of distinct elements of $\mathcal{D}$.

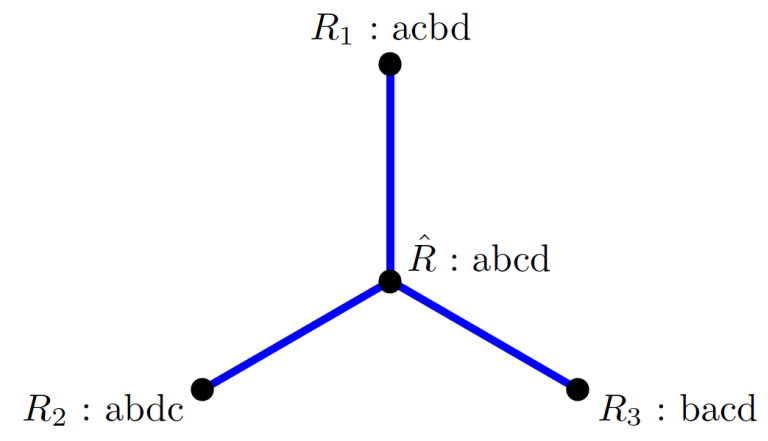

Figure 4: A generalized single-crossing domain

The following result characterizes the single-crossing property and its generalization to trees directly in terms of the structure of the underlying domain, i.e., without explicit reference to the associated graph.

Proposition 4.2. a) A domain $\mathcal{D}$ has the single-crossing property with respect to some linear order on $\mathcal{D}$ if and only if, for all $x, y, z, w \in X$ such that each of the sets $\mathcal{V}_{x y}^{\mathcal{D}}, \mathcal{V}_{y x}^{\mathcal{D}}$, $\mathcal{V}_{z w}^{\mathcal{D}}$, and $\mathcal{V}_{w z}^{\mathcal{D}}$ is non-empty, we have:

$$
\mathcal{V}_{x y}^{\mathcal{D}} \subseteq \mathcal{V}_{z w}^{\mathcal{D}} \text { or } \mathcal{V}_{x y}^{\mathcal{D}} \subseteq \mathcal{V}_{w z}^{\mathcal{D}} .
$$


b) Let $\mathbb{F}=\left\{\mathcal{V}_{x y}^{\mathcal{D}} \mid x, y \in X\right\}$. A domain $\mathcal{D}$ has the generalized single-crossing property if and only if $\mathbb{F}$ has the Helly property and, for all distinct $x, y, z, w \in X$, at least one of the following four sets is empty:

$$
\mathcal{V}_{x y}^{\mathcal{D}} \cap \mathcal{V}_{z w}^{\mathcal{D}}, \mathcal{V}_{x y}^{\mathcal{D}} \cap \mathcal{V}_{w z}^{\mathcal{D}}, \mathcal{V}_{y x}^{\mathcal{D}} \cap \mathcal{V}_{z w}^{\mathcal{D}}, \mathcal{V}_{y x}^{\mathcal{D}} \cap \mathcal{V}_{w z}^{\mathcal{D}} .
$$

Proof. a) Evidently, every single-crossing domain satisfies (4.1). To prove the converse, we notice that this condition allows one to order the family of all sets of the form $\mathcal{V}_{x y}^{\mathcal{D}}$ so that, for an appropriate sequence of pairs of alternatives $\left(a_{1}, b_{1}\right), \ldots,\left(a_{m}, b_{m}\right)$,

$$
\mathcal{V}_{a_{1} b_{1}}^{\mathcal{D}} \subseteq \mathcal{V}_{a_{2} b_{2}}^{\mathcal{D}} \subseteq \ldots \subseteq \mathcal{V}_{a_{m} b_{m}}^{\mathcal{D}} \text { and } \mathcal{V}_{b_{1} a_{1}}^{\mathcal{D}} \supseteq \mathcal{V}_{b_{2} a_{2}}^{\mathcal{D}} \supseteq \ldots \supseteq \mathcal{V}_{b_{m} a_{m}}^{\mathcal{D}}
$$

It is now easily verified that $\mathcal{D}$ has the single-crossing property with respect to any linear order of the members of $\mathcal{D}$ which lists the elements of $\mathcal{V}_{a_{1} b_{1}}^{\mathcal{D}}$ first, then the elements of $\mathcal{V}_{a_{2} b_{2}}^{\mathcal{D}} \backslash \mathcal{V}_{a_{1} b_{1}}^{\mathcal{D}}$ second, and further lists elements of $\mathcal{V}_{a_{j} b_{j}}^{\mathcal{D}} \backslash \mathcal{V}_{a_{j-1} b_{j-1}}^{\mathcal{D}}$ after listing $\mathcal{V}_{a_{j-1} b_{j-1}}^{\mathcal{D}}$.

b) Suppose that $\mathcal{D}$ has the generalized single-crossing property. Then, $\Gamma_{\mathcal{D}}$ is a tree. By Lemma 3.1(iii), the betweenness on $\mathcal{D}$ coincides with the induced geodesic betweenness on $\Gamma_{\mathcal{D}}$. In particular, $\mathcal{D}$ is a median domain, and as such satisfies the Helly property for convex sets, and all elements of $\mathbb{F}$ are convex. To verify (4.2), assume by contradiction that

$$
P \in \mathcal{V}_{x y}^{\mathcal{D}} \cap \mathcal{V}_{z w}^{\mathcal{D}}, Q \in \mathcal{V}_{x y}^{\mathcal{D}} \cap \mathcal{V}_{w z}^{\mathcal{D}}, R \in \mathcal{V}_{y x}^{\mathcal{D}} \cap \mathcal{V}_{z w}^{\mathcal{D}}, S \in \mathcal{V}_{y x}^{\mathcal{D}} \cap \mathcal{V}_{w z}^{\mathcal{D}},
$$

for $P, Q, R, S \in \mathcal{D}$. Consider a shortest path between $P$ and $R$ and a shortest path between $Q$ and $S$. The first path lies entirely in $\mathcal{V}_{z w}^{\mathcal{D}}$ and the second one lies entirely in $\mathcal{V}_{w z}^{\mathcal{D}}$; in particular, they do not intersect. But on each of them there is a switch from $x y$ to $y x$, and thus there exist two pairs of neighboring orders such that one of them is in $\mathcal{V}_{x y}^{\mathcal{D}}$ and the other one in $\mathcal{V}_{y x}^{\mathcal{D}}$. This contradicts the generalized single-crossing property.

Conversely, suppose that a domain $\mathcal{D}$ satisfies the Helly property and condition (4.2). Proposition 2.2 then implies that $\mathcal{D}$ is a median domain, hence by Lemma 3.1(i), the betweenness in $\mathcal{D}$ coincides with the geodesic betweenness in $\Gamma_{\mathcal{D}}$. By $(4.2), \Gamma_{\mathcal{D}}$ is acyclic, hence a tree, which implies the generalized single-crossing property, as desired.

\section{Maximal Condorcet Domains Revisited}

The problem of characterizing maximal Condorcet domains has received considerable attention in the literature. Since every maximal Condorcet domain is closed, our analysis allows one to address this problem by studying the structure of the associated median graph. In particular, we will show that, if the median graph associated with a maximal domain is acyclic, then it must, in fact, be a chain, i.e., among all trees only chains can be associated with maximal Condorcet domains. On the other hand, it is well-known that single-crossing domains are in general not maximal as Condorcet domains (see, e.g., Monjardet [2009]). However, occasionally this happens, and we provide a simple necessary and sufficient condition for a single-crossing domain to be a maximal Condorcet domain. Finally, we demonstrate by means of an example that even though the induced median 
graph of a maximal Condorcet domain is never a tree different from a chain, it does not need to have the structure of a distributive lattice either (in view of Corollary 3.2 above, such maximal Condorcet domain cannot contain two completely reversed orders).

If $X$ has three elements, all maximal Condorcet domains have four elements and are either of the type shown in Figure 2 (connected and single-crossing) or of the type shown to the left of Figure 3 (not connected and cyclic). If $|X|>3$, not all maximal Condorcet domains on $X$ have the same cardinality. Call a domain a maximum Condorcet domain on $X$ if it achieves the largest cardinality among all Condorcet domains on $X$. Evidently, every maximum Condorcet domain is also maximal (and therefore closed), but not vice versa. If $X$ has four elements, the cardinality of a maximum Condorcet domain is known to be 9 , see Figure 5 for a domain attaining this number.

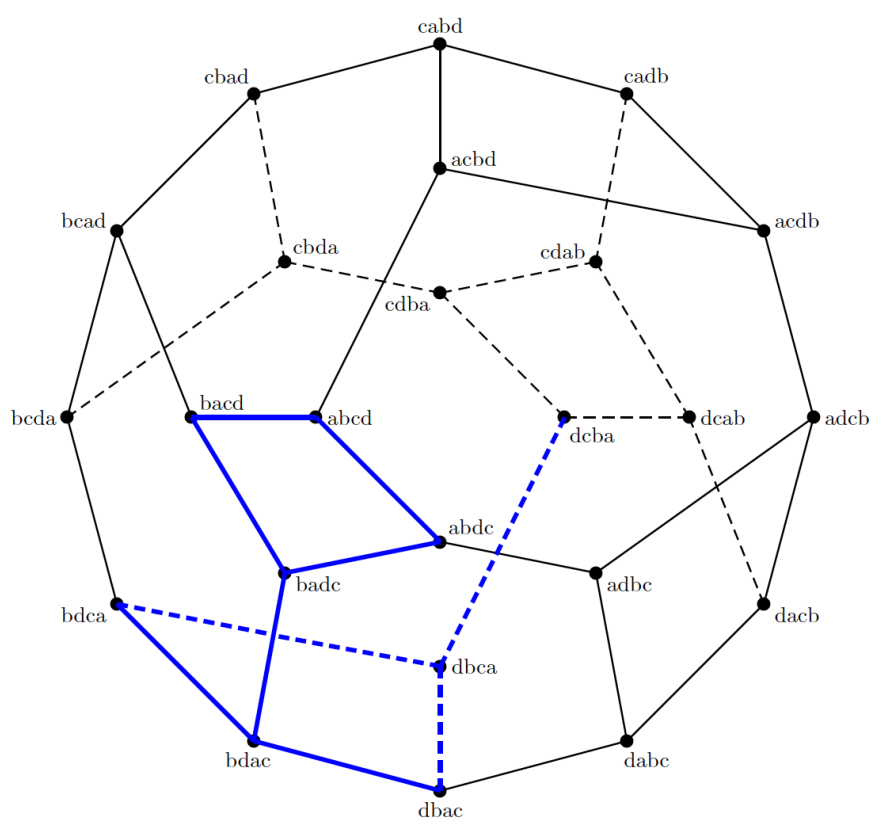

Figure 5: A connected maximum Condorcet domain on $\{a, b, c, d\}$

Note that the domain shown in Figure 5 is connected. Indeed, most of the known general results about the size and the structure of maximal Condorcet domains pertain to connected Condorcet domains; for instance, the domains described in Abello [1991], Chameni-Nembua [1989], Danilov et al. [2012] and Galambos and Reiner [2008] are all connected. However, not all maximal Condorcet domains are connected, as we have already seen above. Figure 6 shows a maximal Condorcet domain with 8 elements on $\{a, b, c, d\}$ that is not connected; in fact, it belongs to the class of the so-called 'symmetric' Condorcet domains studied in Danilov and Koshevoy [2013]; a domain is called symmetric if it contains with any order also its completely reversed order.

All examples of maximal Condorcet domains that we have given so far are either single-crossing domains, or their associated graph contains at least one 4-cycle. ${ }^{11}$ As we

\footnotetext{
${ }^{11}$ It is well-known that every median graph that is not a tree contains at least one 4-cycle; this can be
} 


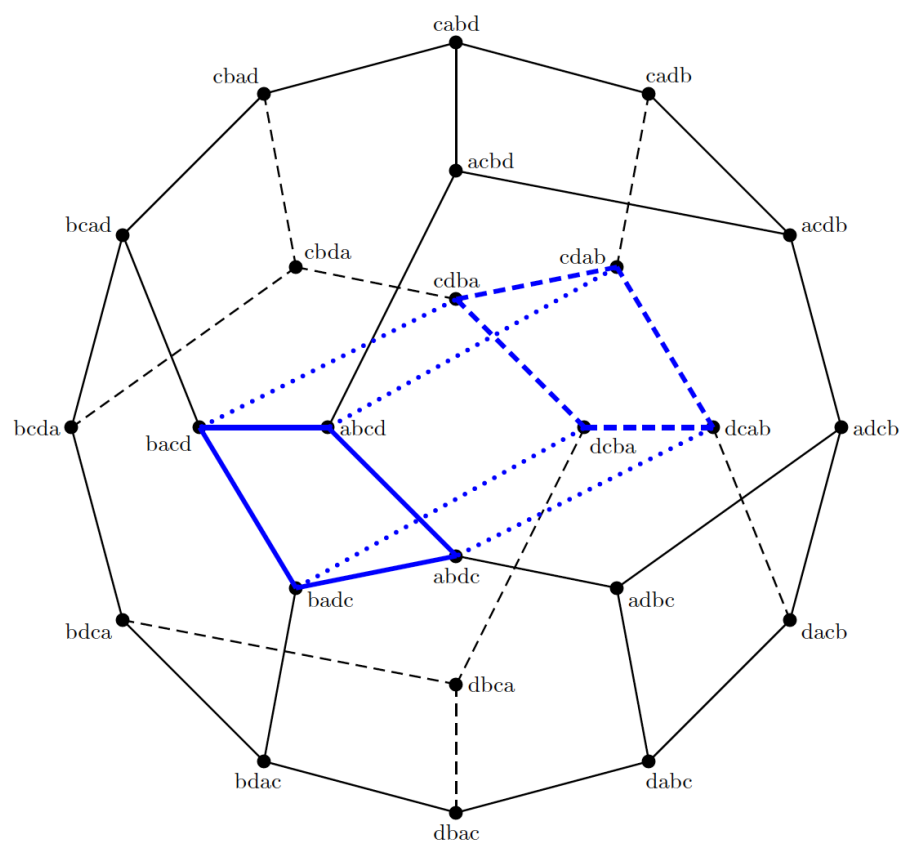

Figure 6: A non-connected maximal Condorcet domain on $\{a, b, c, d\}$

will see, this is a general feature of all maximal Condorcet domains. To show this we need to do some preliminary work.

Lemma 5.1. Suppose $\mathcal{D}$ is generalized single-crossing and $Q R$ is an edge in $\Gamma_{\mathcal{D}}$. Suppose $P \in[Q, R]$ is different from $Q$ and $R$. Then $\mathcal{D}^{\prime}=\mathcal{D} \cup\{P\}$ is also a generalized singlecrossing domain and, in particular, $\mathcal{D}$ is not a maximal Condorcet domain.

Proof. We add $P$ to the graph splitting the edge $Q R$, placing $P$ in the middle. The new graph will be still a tree and the preferences of $\mathcal{D}^{\prime}=\mathcal{D} \cup\{P\}$ will be intermediate with respect to this tree, hence generalized single-crossing. In particular, $\mathcal{D}^{\prime}=\mathcal{D} \cup\{P\}$ is a median domain and hence a closed Condorcet domain by Theorem 2 .

We note that the lemma is specific to trees; if $\Gamma_{\mathcal{D}}$ is a rectangle, the corresponding statement would not hold as the right-hand side of Figure 3 shows.

Corollary 5.1. Any maximal generalized single-crossing domain on $X$ is connected, i.e., is a subgraph of the permutohedron.

Now we can prove the first of the two main results of this section.

Theorem 7. Let $\mathcal{D}$ be a maximal Condorcet domain. If $\Gamma_{\mathcal{D}}$ is a tree, it is, in fact, a chain.

verified as in Footnote 10 above. Note that median graphs can contain also larger cycles (cf.Figure 5) but these can never be 'minimal' (in an appropriate sense); every minimal cycle of any median graph is a 4-cycle. 
Proof. Let $\mathcal{D}$ be a maximal Condorcet domain, and assume that $\Gamma_{\mathcal{D}}$ is a tree but not a chain. Then there exists a vertex $R$ in $\Gamma_{\mathcal{D}}$ of degree at least 3. Consider now any three neighbors of $R$ in $\Gamma_{\mathcal{D}}$, say $R_{1}, R_{2}$ and $R_{3}$. Since, by Corollary 5.1, $\mathcal{D}$ is connected, there are three distinct ordered pairs $\left(x_{i}, y_{i}\right), i=1,2,3$, of alternatives such that $R_{i}=R \backslash\left\{\left(x_{i}, y_{i}\right)\right\} \cup\left\{\left(y_{i}, x_{i}\right)\right\}$. We will say that $R_{i}$ is obtained from $R$ by switching the pair of adjacent alternatives $\left(x_{i}, y_{i}\right)$. Moreover, since in every pair $\left(x_{i}, y_{i}\right), i=1,2,3$, the alternatives are adjacent in $R$, there must exist at least two pairs that have no alternative in common, say $\left\{x_{1}, y_{1}\right\} \cap\left\{x_{2}, y_{2}\right\}=\emptyset$.

Now let $R^{\prime}$ be the order that coincides with $R$ except that both pairs $\left(x_{1}, y_{1}\right)$ and $\left(x_{2}, y_{2}\right)$ in $R^{\prime}$ are switched, i.e., $y_{1} R^{\prime} x_{1}$ and $y_{2} R^{\prime} x_{2}$, and consider the domain $\mathcal{D} \cup\left\{R^{\prime}\right\}$. Since $x_{1}, y_{1}$ and $x_{2}, y_{2}$ are neighbors in each of the orders $R, R_{1}, R_{2}, R^{\prime}$, for every three alternatives $\{a, b, c\}$ no new order among them appears in $R^{\prime}$ which has not yet occurred in $R, R_{1}$, or $R_{2}$. Hence, by Theorem $\left.1 \mathrm{~d}\right), \mathcal{D} \cup\left\{R^{\prime}\right\}$ is a Condorcet domain. By the maximality of $\mathcal{D}$, this implies $R^{\prime} \in \mathcal{D}$. But in this case, the graph $\Gamma_{\mathcal{D}}$ evidently contains the 4-cycle $\left\{R, R_{1}, R^{\prime}, R_{2}\right\}$, contradicting the assumed acyclicity $\Gamma_{\mathcal{D}}$. Hence, there cannot exist a vertex of degree 3 or larger, i.e., $\Gamma_{\mathcal{D}}$ is a chain.

Figure 4 (cf. Example 3 above) illustrates the proof of Theorem 7: one easily verifies that the order badc can be added to the depicted domain $\mathcal{D}$, creating a 4 -cycle in the associated graph $\Gamma_{\mathcal{D}}$; in particular, $\mathcal{D}$ is not maximal.

As we will see some - but by far not all - maximal single-crossing domains are also maximal as Condorcet domains. Figure 5, however, shows instances of non-maximality: the depicted maximal Condorcet domain contains four maximal single-crossing domains as proper subdomains. The next result characterizes exactly when a maximal single-crossing domain is also a maximal Condorcet domain.

To formulate the result, we need the following definitions. Let $\mathcal{D} \subseteq \mathcal{R}(X)$ be a maximal single-crossing domain and $|X|=n$. Then we know that the associated graph $\Gamma_{\mathcal{D}}$ is linear. By Corollary 5.1, it is a subgraph of the permutohedron. Let us enumerate orders of $\mathcal{D}$ so that the edges of $\Gamma_{\mathcal{D}}$ are $R_{1} R_{2}, R_{2} R_{3}, \ldots, R_{m-1} R_{m}$. Then the sequence $\left\langle R_{1}, \ldots, R_{m}\right\rangle$ will be called a maximal chain. Due to Lemma 5.1, each edge $R_{i} R_{i+1}$ in $\Gamma_{\mathcal{D}}$ has a unique switching pair of alternatives $\left(x_{i}, y_{i}\right)$ which are adjacent in $R_{i}$. Due to the (standard) single-crossing condition, once switched the pair is never switched back, so for a two-element subset of alternatives $\{x, y\}$ we cannot have both pairs $(x, y)$ and $(y, x)$ as switching pairs. Moreover, $R_{1}$ and $R_{m}$ are completely reversed. Indeed, if $R_{m}$ is not $R_{1}$, which is completely reversed $R_{1}$, then we can add $\underline{R_{1}}$ at the end of the sequence $\left\langle R_{1}, \ldots, R_{m}\right\rangle$ and add another vertex to our graph in contradiction to maximality of $\mathcal{D}$. Hence, $m=\frac{(n-1) n}{2}+1$, so, in particular, all maximal chains are of the same length.

Without loss of generality we may assume that $X=\{1,2, \ldots, n\}$, that $\bar{R}=R_{1}=$ $12 \ldots n-1 n$ and that $\underline{R}=R_{m}=n n-1 \ldots 21$. Traveling along the maximal chain $\left\langle R_{1}, \ldots, R_{m}\right\rangle$ from $\bar{R}$ in direction of $\underline{R}$ the alternatives in every 2-element subset $\{x, y\}$, where $x<y$, switch exactly once from $x y$ to $y x$. That is, for each pair $(x, y) \in X \times X$ there exists exactly one edge $\left(R_{j}, R_{j+1}\right)$ such that $x R_{1} y, \ldots, x R_{j} y$ and $y R_{j+1} x, \ldots, y R_{m} x$. We say that this maximal chain satisfies the pairwise concatenation condition if the switching 


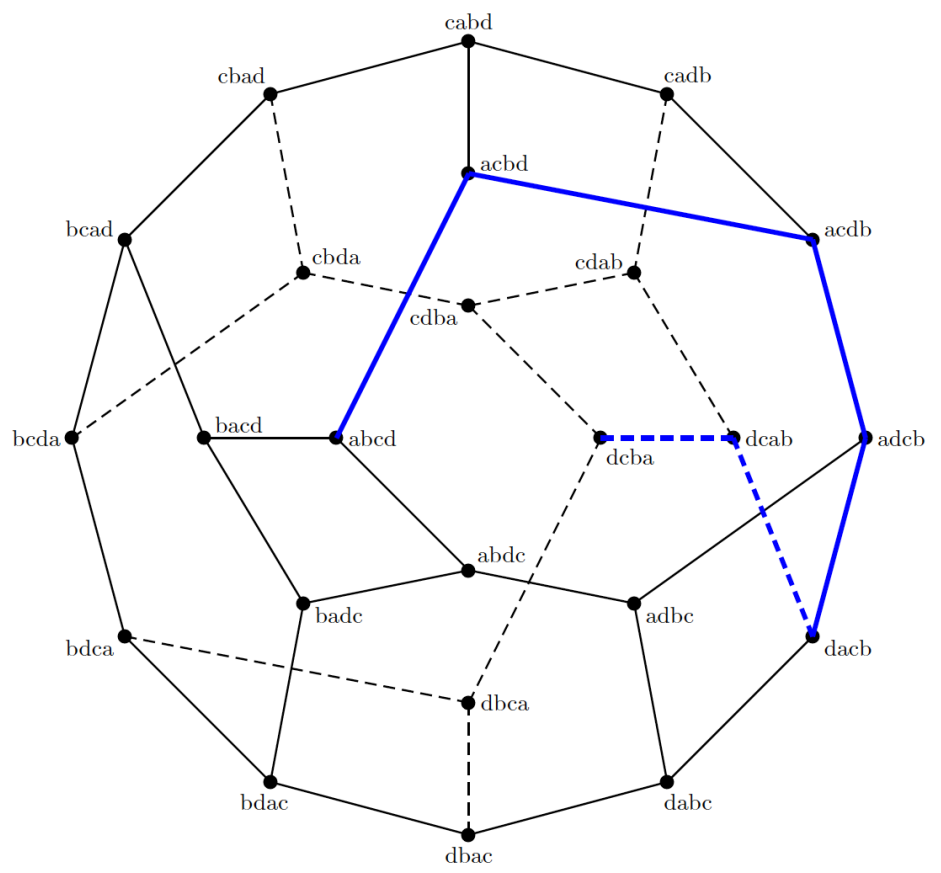

Figure 7: A maximal chain constituting a maximal Condorcet domain on $\{a, b, c, d\}$

pairs corresponding to any two adjacent edges have one alternative in common. Formally, a maximal chain $\left\langle R_{1}, \ldots, R_{m}\right\rangle$ with the switching pairs $\left(x_{j}, y_{j}\right)$ of the edges $R_{j} R_{j+1}$, $j=1, \ldots, m-1$, satisfies the pairwise concatenation condition if

$$
\left\{x_{j}, y_{j}\right\} \cap\left\{x_{j+1}, y_{j+1}\right\} \neq \emptyset
$$

for all $j=1, \ldots, m-1$. Note that the intersection in (5.1) then has exactly one element. We note that the sequence of switching pairs $\left(x_{1}, y_{1}\right), \ldots,\left(x_{m-1}, y_{m-1}\right)$ determines the maximal chain $\left\langle R_{1}, \ldots, R_{m}\right\rangle$ uniquely.

To illustrate the pairwise concatenation condition consider Figure 7 which depicts a single-crossing domain on $X=\{a, b, c, d\}$ with $m=7$. The maximal chain for this domain is:

$$
R_{1}=a b c d, R_{2}=a c b d, R_{3}=a c d b, R_{4}=a d c b, R_{5}=d a c b, R_{6}=d c a b, R_{7}=d c b a,
$$

with the sequence of switching pairs $(b, c),(b, d),(c, d),(a, d),(a, c),(a, b)$, which obviously satisfies the pairwise concatenation condition.

We will now show that the pairwise concatenation condition is necessary and sufficient for a maximal single-crossing domain to be a maximal Condorcet domain. ${ }^{12}$

Definition 3 (Galambos and Reiner [2008]). Two maximal chains are equivalent if their respective sequences of switching pairs can be transformed one to another by swapping adjacent non-intersecting pairs.

\footnotetext{
${ }^{12}$ As we learned after completion of the first version of the present paper, Bernard Monjardet found the same condition in his unpublished lecture notes Monjardet [2007] (without proof).
} 
It is easily verified that this notion indeed defines an equivalence relation on the class of all maximal chains. To illustrate the definition, consider the maximum Condorcet domain $\mathcal{D}$ on the set $X=\{a, b, c, d\}$ depicted in Figure 5 above. The following sequence is one of its maximal chains:

$$
\mathcal{C}_{1}:=\langle a b c d, a b d c, b a d c, b d a c, d b a c, d b c a, d c b a\rangle
$$

with the corresponding sequence of switching pairs

$$
(d, c),(a, b),(a, d),(b, d),(a, c),(b, c) .
$$

If one swaps the first two pairs in this sequence, i.e. $(d, c)$ and $(a, c)$, one obtains the sequence of switching pairs corresponding to the equivalent maximal chain:

$$
\mathcal{C}_{2}:=\langle a b c d, b a c d, b a d c, b d a c, d b a c, d b c a, d c b a\rangle,
$$

which is also contained in the maximum domain $\mathcal{D}$. If in the sequence $(5.2)$, one switches the fourth and fifth pair, i.e. $(b, d)$ and $(a, c)$, one obtains another equivalent maximal chain of $\mathcal{D}$ :

$$
\mathcal{C}_{3}:=\langle a b c d, a b d c, b a d c, b d a c, b d c a, d b c a, d c b a\rangle .
$$

Finally, if one switches both pairs in $(5.2)$, first $(d, c)$ against $(a, b)$ and then $(b, d)$ against $(a, c)$, one obtains the equivalent maximal chain:

$$
\mathcal{C}_{4}:=\langle a b c d, b a c d, b a d c, b d a c, b d c a, d b c a, d c b a\rangle .
$$

Evidently, the maximum Condorcet domain $\mathcal{D}$ is the union of the pairwise equivalent maximal chains $\mathcal{C}_{1}, \mathcal{C}_{2}, \mathcal{C}_{3}, \mathcal{C}_{4}$ that it contains. The following result due to [Galambos and Reiner, 2008, Th. 2] shows that this is a general property of all maximal Condorcet domains that contain at least one maximal chain.

Theorem 8 (Galambos and Reiner [2008]). Let $\mathcal{D}$ be a single-crossing domain and let $\left\langle R_{1}, \ldots, R_{m}\right\rangle$ be its corresponding maximal chain. Then the maximal Condorcet domain containing $\mathcal{D}$ consists of all linear orders of all maximal chains equivalent to $\left\langle R_{1}, \ldots, R_{m}\right\rangle$.

We can now state the second main result of this section.

Theorem 9. A maximal single-crossing domain $\mathcal{D} \subseteq \mathcal{R}(X)$ is a maximal Condorcet domain if and only if the maximal chain corresponding to $\mathcal{D}$ satisfies the pairwise concatenation condition.

Proof. Let $\mathcal{D}$ be a maximal single-crossing domain and $\left\langle R_{1}, \ldots, R_{m}\right\rangle$ be the corresponding maximal chain. To verify the necessity of the pairwise concatenation condition suppose, by contraposition, that for two consecutive switching pairs $\left(x_{j}, y_{j}\right)$ and $\left(x_{j+1}, y_{j+1}\right)$ one has $\left\{x_{j}, y_{j}\right\} \cap\left\{x_{j+1}, y_{j+1}\right\}=\emptyset$. Without loss of generality assume that $x_{j} R_{j} y_{j}$ and $y_{j} R_{j+1} x_{j}$, as well as $x_{j+1} R_{j+1} y_{j+1}$ and $y_{j+1} R_{j+2} x_{j+1}$. Since $\left\{x_{j}, y_{j}\right\} \cap\left\{x_{j+1}, y_{j+1}\right\}=\emptyset$, both pairs of alternatives $\left(x_{j}, y_{j}\right)$ and $\left(x_{j+1}, y_{j+1}\right)$ must be adjacent in all three orderings $R_{j}, R_{j+1}, R_{j+2}$, respectively. In particular, these three orders agree in the ranking of all pairs except for 


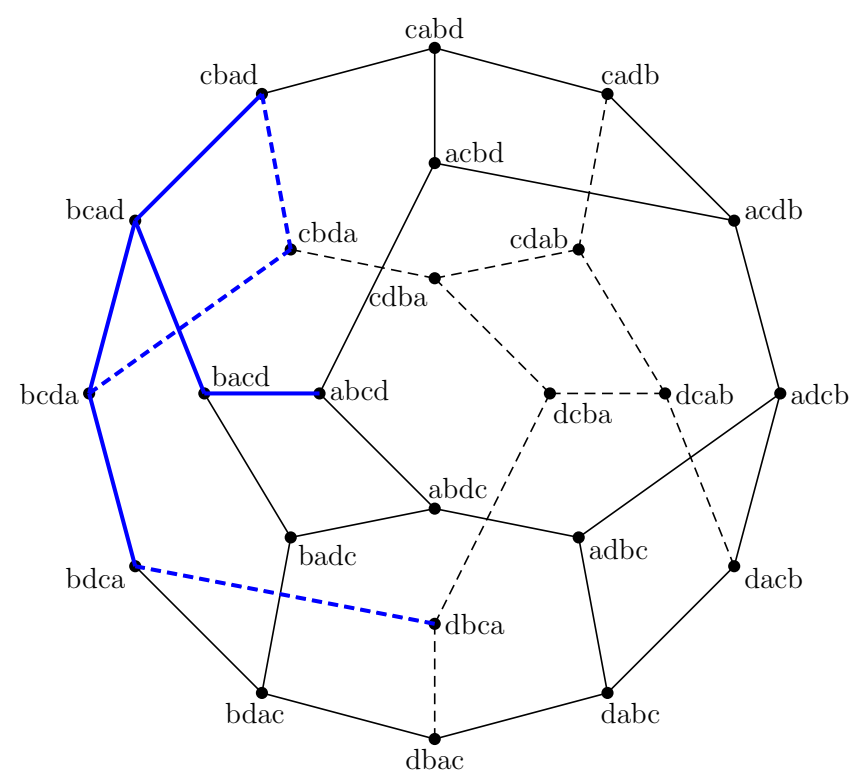

Figure 8: A maximal Condorcet domain with no pair of completely reversed orders

the two pairs $\left(x_{j}, y_{j}\right)$ and $\left(x_{j+1}, y_{j+1}\right)$, respectively. Consider the order $R^{\prime}$ that has $x_{j} R^{\prime} y_{j}$ and $y_{j+1} R^{\prime} x_{j+1}$ and agrees with each of $R_{j}, R_{j+1}, R_{j+2}$ in the ranking of all other pairs. Clearly, $R^{\prime} \notin \mathcal{D}$, but the restriction of $R^{\prime}$ to any triple of distinct alternatives coincides with the restriction of $R_{j}, R_{j+1}$, or $R_{j+2}$ to this triple. Hence, $\mathcal{D} \cup\left\{R^{\prime}\right\}$ is a Condorcet domain by Theorem $1 \mathrm{~d}$ ), and thus $\mathcal{D}$ is not maximal. This demonstrates the necessity of the pairwise concatenation condition.

The sufficiency of the pairwise concatenation condition follows from Theorem 8. Indeed, it is easily seen that a maximal chain satisfying the pairwise concatenation condition has no other maximal chain equivalent to it, i.e. it forms an equivalence class of its own. By Theorem 8, the corresponding maximal single-crossing domain is a maximal Condorcet domain.

In light of Theorem 7 and Corollary 3.2 above, a natural conjecture is that all maximal Condorcet domains are distributive lattices. However, this turns out not to be true, as the example in the following figure shows. The domain depicted in Figure 8 is connected and maximal but not a distributive lattice; in view of Corollary 3.2 it clearly cannot contain a pair of completely reversed orders.

It seems to be an interesting and worthwhile subject for future research to characterize the class of median graphs that can arise as the associated graphs of maximal Condorcet domains. However, this approach has to be complemented by other considerations as well, since the graph alone does in general not contain information about maximality. For instance, we have already seen that chains may be the associated graphs of maximal Condorcet domains only if their length is one greater than a triangular number, i.e., $\frac{1}{2}(n-1) n+1$. Another example that illustrates this point quite drastically is the 4-cycle. 
We have already seen above that even non-median domains can induce a 4-cycle, albeit the betweenness structures of the domain and the 4-cycle will not be the same (see the domain in the middle of Figure 3). But even if we insist on them being isomorphic, thus ensuring that the corresponding domain is a closed Condorcet domain, the 4-cycle may or may not yield a maximal Condorcet domain. For instance, all 4-cycles in Figures 5, 6 and 8 evidently do not correspond to maximal Condorcet domains since they form proper subdomains. But Figure 9 shows that the 4-cycle can also be the associated graph of a maximal domain in $\mathcal{R}(\{a, b, c, d\})$. Such maximal domain of size 4 can be found in $\mathcal{R}(X)$ for any cardinality of $X$, as shown by Danilov and Koshevoy [2013].

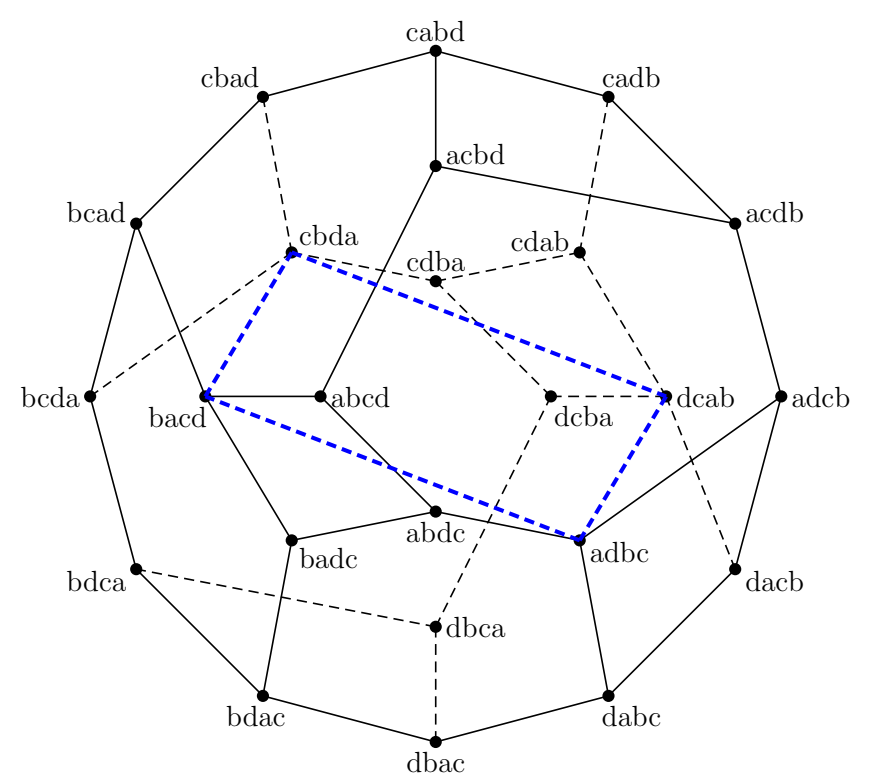

Figure 9: A maximal Condorcet domain with four elements

\section{Arrovian Aggregation and Strategy-Proof Social Choice on Median Preference Domains}

Closed Condorcet domains not only preclude intransitivities in pairwise majority voting, they are also endowed with a large class of further aggregation rules satisfying Arrow's independence condition. This follows from the analysis of Nehring and Puppe [2007, 2010]. Indeed, their main result entails a characterization of all Arrovian aggregators on such domains under an additional monotonicity condition. In the first subsection below, we apply their result to describe the class of all monotone Arrovian aggregators on closed Condorcet domains. The monotonicity condition plays then a crucial role in the construction of strategy-proof social choice functions on such domains in the second subsection. 


\subsection{Characterization of all Arrovian Aggregators}

Let $N=\{1,2, \ldots, n\}$ be the set of voters. An aggregator on a domain $\mathcal{D} \subseteq \mathcal{R}(X)$ is a mapping $f: \mathcal{D}^{n} \rightarrow \mathcal{D}$ that assigns an order in $\mathcal{D}$ to each profile of individual orders in $\mathcal{D}$. The following conditions on aggregators have been extensively studied in the literature.

Full Range. For all $R \in \mathcal{D}$, there exist $R_{1}, \ldots, R_{n}$ such that $f\left(R_{1}, \ldots, R_{n}\right)=R$.

Unanimity. For all $R \in \mathcal{D}, f(R, \ldots, R)=R$.

Independence. For all $R_{1}, \ldots, R_{n}, R_{1}^{\prime}, \ldots, R_{n}^{\prime} \in \mathcal{D}$ and all pairs of distinct alternatives $x, y \in X$, if $x R y$ and, for all $i \in N,\left[x R_{i} y \Leftrightarrow x R_{i}^{\prime} y\right]$, then $x R^{\prime} y$, where $R=f\left(R_{1}, \ldots, R_{n}\right)$ and $R^{\prime}=f\left(R_{1}^{\prime}, \ldots, R_{n}^{\prime}\right)$.

An aggregator is called Arrovian if it satisfies unanimity and independence. In what follows, we will be concerned with Arrovian aggregators that satisfy in addition the following monotonicity condition.

Monotonicity For all $R_{1}, \ldots, R_{n}, R_{i}^{\prime} \in \mathcal{D}$ and all pairs of distinct alternatives $x, y$, if $x R^{\prime} y$ and $x R_{i} y$, then $x R y$, where $R^{\prime}=f\left(R_{1}, \ldots, R_{i}^{\prime}, \ldots, R_{n}\right)$ and $R=f\left(R_{1}, \ldots, R_{i}, \ldots, R_{n}\right)$.

The monotonicity condition can be rephrased as follows. An aggregator $f$ is monotone if and only if, for all $R_{i}, R_{i}^{\prime} \in \mathcal{D}$ and all $R_{-i} \in \mathcal{D}^{n-1}$,

$$
f\left(R_{i}, R_{-i}\right) \in\left[R_{i}, f\left(R_{i}^{\prime}, R_{-i}\right)\right] .
$$

This has the following interpretation. Consider any pair of distinct alternatives $a$ and $b$, and suppose that $a R_{i} b$ according to agent $i$ 's true order $R_{i}$. Then, if agent $i$ can force the social order to rank $a$ above $b$ by submitting some order $R_{i}^{\prime}$, the social order would also rank $a$ above $b$ if agent $i$ submitted his true preference $R_{i}$. In other words, no agent can benefit in a pairwise comparison from any misrepresentation. The monotonicity condition thus has a clear 'non-manipulability' flavor which we will further exploit.

The conjunction of independence and monotonicity is equivalent to the following single condition.

Monotone Independence For all $R_{1}, \ldots, R_{n}$ and for all pairs $(x, y)$ of distinct alternatives $x, y \in X$, if $x R y$, where $R=f\left(R_{1}, \ldots, R_{n}\right)$ and $\left[a R_{i} b \Rightarrow a R_{i}^{\prime} b\right]$ for all $i \in N$, then $a R^{\prime} b$, where $R^{\prime}=f\left(R_{1}^{\prime}, \ldots, R_{n}^{\prime}\right)$.

Note also that under monotonicity, unanimity can be deduced from the full range condition, i.e., from the assumption that the aggregator is onto.

For every pair $(x, y) \in X \times X$ of distinct alternatives, let $\mathcal{W}_{x y}$ be a non-empty collection of non-empty subsets $W \subseteq N$ of voters (the winning coalitions for $x$ against $y$ ) satisfying

$$
\left[W \in \mathcal{W}_{x y} \text { and } W^{\prime} \supseteq W\right] \Rightarrow W^{\prime} \in \mathcal{W}_{x y} .
$$

Definition 4. A collection $\mathcal{W}=\left\{\mathcal{W}_{a b} \mid(a, b) \in X \times X, a \neq b\right\}$ is called a structure of winning coalitions if, for all distinct pairs of alternatives $(x, y) \in X \times X$,

$$
W \in \mathcal{W}_{x y} \Leftrightarrow W^{c} \notin \mathcal{W}_{y x},
$$

where $W^{c}=N \backslash W$ denotes the complement of the coalition $W$. 
Examples. There are numerous examples of structures of winning coalitions. The simplest are the dictatorships which arise whenever there exists an individual $i$ such that, for all distinct pairs $(x, y)$, a coalition $W$ belongs to $\mathcal{W}_{x y}$ if and only if $i \in W$. More generally, an oligarchy is characterized by the existence of a non-empty set $M$ of voters such that, for all distinct pairs $(x, y)$, either (i) $W \in \mathcal{W}_{x y} \Leftrightarrow M \subseteq W$, or (ii) $W \in \mathcal{W}_{y x} \Leftrightarrow M \subseteq W$. Note that, by (6.3), if (i) holds, then $\{i\} \in \mathcal{W}_{y x}$ for all $i \in M$; similarly, if (ii) holds, then $\{i\} \in \mathcal{W}_{x y}$ for all $i \in M$.

A structure of winning coalitions is anonymous if, for any fixed pair $(x, y) \in X \times X$, membership of a coalition $W$ to $\mathcal{W}_{x y}$ depends only on the number of individuals in $W$. A special case is pairwise majority voting which requires that for all pairs $(x, y) \in X \times X$ of distinct alternatives

$$
W \in \mathcal{W}_{x y} \Leftrightarrow|W|>n / 2
$$

Note that this is jointly satisfiable with (6.3) only if $n$ is odd.

Given a domain $\mathcal{D} \in \mathcal{R}(X)$, a structure of winning coalitions is said to be order preserving on $\mathcal{D}$ if, for every pair of distinct alternatives $(x, y) \in X \times X$ and every pair of distinct alternatives $(z, w) \in X \times X$,

$$
\mathcal{V}_{x y}^{\mathcal{D}} \subseteq \mathcal{V}_{z w}^{\mathcal{D}} \Rightarrow \mathcal{W}_{x y} \subseteq \mathcal{W}_{z w}
$$

Observe that pairwise majority voting always defines an order preserving structure of winning coalitions ( $\operatorname{since} \mathcal{W}_{x y}=\mathcal{W}_{z w}$ for all distinct $x, y$ and $z, w$ in the case of pairwise majority voting).

The following result is due to Nehring and Puppe [2007].

Proposition 6.1. Let $\mathcal{D}$ be a closed Condorcet domain and let $\mathcal{W}$ be any order preserving structure of winning coalitions on $\mathcal{D}$. For all preference profiles $\left(R_{1}, \ldots, R_{n}\right) \in \mathcal{D}^{n}$ there exists a unique order $R^{*} \in \mathcal{D}$ such that, for every pair of distinct alternatives $(x, y) \in X \times X$

$$
x R^{*} y \Leftrightarrow\left\{i \in N \mid x R_{i} y\right\} \in \mathcal{W}_{x y} .
$$

The aggregator defined by (6.5) is a monotone Arrovian aggregator. Conversely, every monotone Arrovian aggregator on $\mathcal{D}$ takes this form for some order preserving structure of winning coalitions $\mathcal{W}$.

Proof. The proof can be deduced from [Nehring and Puppe, 2007, Prop. 3.4]. For the sake of the paper being self-contained, we reproduce the argument here, again emphasizing the role of the Helly property on median domains as in the proof of Theorem 2 above.

For any profile $\left(R_{1}, \ldots, R_{n}\right) \in \mathcal{D}^{n}$, define a binary relation $R^{*}$ by (6.5). First, we show that $R^{*} \in \mathcal{D}$. Consider distinct $x, y \in X$ and distinct $z, w \in X$ such that $x R^{*} y$ and $z R^{*} w$; we will show that $\mathcal{V}_{x y}^{\mathcal{D}} \cap \mathcal{V}_{z w}^{\mathcal{D}} \neq \emptyset$. By contradiction, suppose that this does not hold, then we would obtain $\mathcal{V}_{x y}^{\mathcal{D}} \subseteq \mathcal{V}_{w z}^{\mathcal{D}}$, and in particular also $\left\{i \in N \mid x R_{i} y\right\} \subseteq\left\{i \in N \mid w R_{i} z\right\}$. At the same time, we would have $\mathcal{W}_{x y} \subseteq \mathcal{W}_{w z}$ because the structure of winning coalitions is order preserving. Thus, $\left\{i \in N \mid x R_{i} y\right\} \in \mathcal{W}_{w z}$, hence also $\left\{i \in N \mid w R_{i} z\right\} \in \mathcal{W}_{w z}$. But the latter contradicts $z R^{*} w$ using (6.3). Thus, the collection of convex sets $\left\{\mathcal{V}_{x y}^{\mathcal{D}} \mid x R^{*} y\right\}$ has 
pairwise non-empty intersections. By the Helly property for convex sets in any Condorcet domain (Proposition 2.2), we obtain

$$
\bigcap_{\left\{x R^{*} y\right\}} \mathcal{V}_{x y}^{\mathcal{D}} \neq \emptyset
$$

which means that $R^{*}$ is an element of $\mathcal{D}$. Thus, (6.5) indeed defines a mapping from $\mathcal{D}^{n}$ to $\mathcal{D}$. It is easily verified that it is onto, monotone and independent, i.e., an Arrovian aggregator.

Conversely, let $f$ be a monotone Arrovian aggregator. By the monotonicity and independence conditions, it is easily seen that $f$ can be defined in terms of a structure of winning coalitions as in (6.5). We thus have only to verify that the corresponding structure of winning coalitions must be order preserving. Thus, assume that, for distinct $(x, y) \in X \times X$ and distinct $(z, w) \in X \times X$, we have $\mathcal{V}_{x y}^{\mathcal{D}} \subseteq \mathcal{V}_{z w}^{\mathcal{D}}$, and $W \in \mathcal{W}_{x y}$. Then, if the profile $\left(R_{1}, \ldots, R_{n}\right)$ is such that all voters in $W$ prefer $x$ to $y$, we must have $x R y$ where $R=f\left(R_{1}, \ldots, R_{n}\right)$. By assumption, all orders in $\mathcal{D}$ which rank $x$ above $y$ must also rank $z$ above $w$, hence $z R w$. By independence, this holds for all profiles in which the agents in $W$ rank $x$ above $y$, hence also $z$ above $w$, i.e., the agents in $W$ are also winning for $z$ versus $w$.

\subsection{Strategy-Proof Social Choice}

It is well-known that on domains on which pairwise majority voting with an odd number of voters is transitive, choosing the Condorcet winner yields a strategy-proof social choice function (see, e.g., Lemma 10.3 in Moulin [1988]). In this final subsection, we use Proposition 6.1 and property (6.1) which is entailed by monotonicity to construct a rich class of further strategy-proof social choice functions on any closed Condorcet domain.

A social choice function $F$ that maps every profile $\left(R_{1}, \ldots, R_{n}\right) \in \mathcal{D}^{n}$ to an element $F\left(R_{1}, \ldots, R_{n}\right) \in X$ is strategy-proof if, for all $i \in N$, all $R_{i}, R_{i}^{\prime} \in \mathcal{D}$ and all $R_{-i} \in \mathcal{D}^{n-1}$,

$$
\left[F\left(R_{i}, R_{-i}\right)\right] R_{i}\left[F\left(R_{i}^{\prime}, R_{-i}\right)\right]
$$

i.e., if no voter can benefit by misrepresenting her true preferences.

For each order $R \in \mathcal{R}(X)$ denote by $\tau(R) \in X$ the top-ranked element of $R$. Let $\mathcal{D} \subseteq \mathcal{R}(X)$ be any closed Condorcet domain, and consider any order preserving structure of winning coalitions $\mathcal{W}$. For every profile $\left(R_{1}, \ldots, R_{n}\right) \in \mathcal{D}^{n}$ let $R_{\mathcal{W}} \in \mathcal{D}$ be the unique order satisfying $(6.5)$ for all distinct $x, y \in X$. Define a social choice function $F_{\mathcal{W}}: \mathcal{D}^{n} \rightarrow X$ by

$$
F_{\mathcal{W}}\left(R_{1}, \ldots, R_{n}\right)=\tau\left(R_{\mathcal{W}}\right)
$$

Theorem 10. Let $\mathcal{D} \subseteq \mathcal{R}(X)$ be any closed Condorcet domain. For every order preserving structure of winning of coalitions $\mathcal{W}$, the social choice function $F_{\mathcal{W}}$ defined by (6.6) is strategy-proof.

Proof. By Proposition 6.1, the aggregator $f_{\mathcal{W}}: \mathcal{D}^{n} \rightarrow \mathcal{D}$ that maps any profile $\left(R_{1}, \ldots, R_{n}\right)$ to the social order $R_{\mathcal{W}}$ according to $(6.5)$ is a monotone Arrovian aggregator; in particular, it satisfies (6.1). In other words, if we denote $R_{\mathcal{W}}=f_{\mathcal{W}}\left(R_{i}, R_{-i}\right)$ 
and $R_{\mathcal{W}}^{\prime}=f_{\mathcal{W}}\left(R_{i}^{\prime}, R_{-i}\right)$, we have for all $R_{1}, \ldots, R_{n}$, all $i$, all $R_{i}^{\prime}$, and all distinct pairs of alternatives $(x, y) \in X \times X$,

$$
\left[x R_{i} y \text { and } x R_{\mathcal{W}}^{\prime} y\right] \Rightarrow x R_{\mathcal{W}} y
$$

This implies at once the strategy-proofness of $F_{\mathcal{W}}$, by contraposition. Indeed, suppose that agent $i$ could benefit by misreporting $R_{i}^{\prime}$, i.e., suppose that $\tau\left(R_{\mathcal{W}}^{\prime}\right) R_{i} \tau\left(R_{\mathcal{W}}\right)$, where $R_{i}$ is agent $i$ 's true preference order. Then, since $\tau\left(R_{\mathcal{W}}^{\prime}\right)$ is the top element of the order $R_{\mathcal{W}}^{\prime}$ we obtain from (6.7), $\tau\left(R_{\mathcal{W}}^{\prime}\right) R_{\mathcal{W}} \tau\left(R_{\mathcal{W}}\right)$. Since $\tau\left(R_{\mathcal{W}}\right)$ is the top element of $R_{\mathcal{W}}$ this implies $\tau\left(R_{\mathcal{W}}^{\prime}\right)=\tau\left(R_{\mathcal{W}}\right)$, i.e., the misrepresentation does not change the chosen alternative.

In case of the classical single-crossing property, the anonymous social choice functions defined by (6.6) are exactly the ones identified by Saporiti [2009]. Thus, in this special case, the class of anonymous social choice functions considered in Theorem 10 exhausts all anonymous strategy-proof social choice functions. It is an open and interesting question whether this holds more generally on all closed Condorcet domains (and whether the anonymity assumption is really necessary for this conclusion).

\section{Appendix: Remaining Proofs}

Lemma 3.1 is proven using a result by Bandelt and Chepoi [1996]. The statement of this result requires some additional definitions, in particular the notion of a 'geometric interval operator,' as follows. An interval operator on a (finite) set $V$ is a mapping that assigns to each pair $(v, w) \in V \times V$ a non-empty subset $[v, w] \subseteq V$, the interval spanned by $v$ and $w$, such that, for all $v, w \in V, v \in[v, w]$ and $[v, w]=[w, v]$. An interval operator is called geometric if it satisfies in addition the following properties. For all $t, u, v, w \in V$,

$$
\begin{aligned}
{[v, v] } & =\{v\}, \\
u \in[v, w] & \Rightarrow[v, u] \subseteq[v, w], \\
t, u \in[v, w] \& t \in[v, u] & \Rightarrow u \in[t, w] .
\end{aligned}
$$

A pair $v, w$ is called an edge if $v \neq w$ and $[v, w]=\{v, w\}$. These edges form a graph $\Gamma$ on the vertex set $V$.

Lemma A.1. Consider a geometric interval operator on $V$, and let $u \in[v, w]$. Then, there exist pairwise distinct $t_{1}, \ldots, t_{m} \in[v, w]$ such that $t_{1}=v, t_{m}=w, t_{k}=u$ for some $k \in\{1, \ldots, m\}$, and such that

$$
\left[t_{1}, t_{2}\right] \subset\left[t_{1}, t_{3}\right] \subset \ldots \subset\left[t_{1}, t_{m}\right]
$$

forms a maximal chain. In particular, the graph induced by a geometric interval operator is connected. 
Proof. The existence of a maximal chain of the required form follows at once from condition (A.2). The pairs $t_{k} t_{k+1}$ must form an edge for $k=1, \ldots m-1$ by maximality of the chain. Thus, any two vertices are connected by a path.

An interval operator is called graphic if, for all $u, v, w \in V, u \in[v, w]$ if and only if $u$ is geodesically between $v$ and $w$ in the induced graph $\Gamma$; note that this is exactly condition (3.1) in Lemma 3.1 above. An interval operator is said to satisfy the triangle condition if, for all triples $u, v, w \in V$ such that

$$
[u, v] \cap[v, w]=\{v\} \text { and }[v, w] \cap[w, u]=\{w\} \text { and }[w, u] \cap[u, v]=\{u\}
$$

all three intervals are edges whenever one of them is. Observe that (A.4) can be satisfied only if either all three elements $u, v, w$ coincide, or are pairwise distinct. The following result is due to [Bandelt and Chepoi, 1996, Th.1].

Proposition A.1. Any geometric interval operator satisfying the triangle condition is graphic.

We want to apply Proposition A.1 to prove Lemma 3.1. In order to do so, we first verify the geometricity of the natural interval operator induced by every subdomain of orders.

Lemma A.2. For any domain $\mathcal{D} \subseteq \mathcal{R}(X)$, the interval operator that assigns to every pair $R, R^{\prime} \in \mathcal{D}$ the interval $\left[R, R^{\prime}\right] \cap \mathcal{D}$ is geometric.

Proof. Properties (A.1) and (A.2) are easily verified. To verify the so-called inversion law' (A.3), consider $T, U, V, W \in \mathcal{D}$ as required in the antecedent of (A.3). Let $x, y \in X$ be such that $x T y$ and $x W y$. We have to show that then $x U y$. Since $x, y$ were arbitrarily chosen, this would imply $U \in[T, W]$, as desired. Assume, by contradiction, that $y U x$; then, we must have $x V y$ since by assumption $T \in[V, U]$. But this contradicts the assumption that $U \in[V, W]$.

The following proof of Lemma 3.1 shows that the triangle condition is a powerful sufficient condition for an interval operator to be graphic, since it is indeed satisfied in all three cases considered in Lemma 3.1 (sometimes vacuously).

Proof of Lemma 3.1. (i) In case of a median domain, the triangle condition is vacuously satisfied, since there can obviously be no triples of pairwise distinct elements satisfying (A.4) by the median property. By Proposition A.1 the equivalence (3.1) is satisfied for any median domain.

(ii) Next consider any connected domain $\mathcal{D} \subseteq \mathcal{R}(X)$. There can exist triples satisfying (A.4), but we shall show that in this case none of the three intervals can form an edge, hence the triangle condition is again satisfied. Thus, suppose that the three pairwise distinct orders $U, V, W \in \mathcal{D}$ satisfy (A.4) and, by contradiction, that one of the three intervals is an edge, say $[U, V]=\{U, V\}$. Since $\mathcal{D}$ is connected, there exists $x, y \in X$ such that $U$ and $V$ differ only in the ranking of $x$ versus $y$, say $x U y$ and $y V x$, while $U$ and $V$ agree in the ranking of all other pairs of alternatives. There are two possibilities: either 
$x W y$ or $y W x$. In the first case, we have $U \in[V, W]$ and hence $([U, V] \cap[V, W]) \supseteq\{U, V\}$; in the second case, $V \in[W, U]$ and hence $([W, U] \cap[U, V]) \supseteq\{U, V\}$. In both cases, we thus obtain a contradiction to assumption (A.4). By Proposition A.1 the equivalence (3.1) is satisfied for $\mathcal{D}$.

(iii) Finally, assume that $\mathcal{D}$ is such that $\Gamma_{\mathcal{D}}$ is acyclic, i.e., a tree. As in part (i), we show that there cannot exist triples satisfying (A.4) hence again the triangle condition is satisfied vacuously. ${ }^{13}$ Assume, by way of contradiction, that the pairwise distinct orders $U, V, W \in \mathcal{D}$ satisfy (A.4). By Lemma A.1, there exists a path $\pi_{U V}$ in $\Gamma_{\mathcal{D}}$ connecting $U$ and $V$ that stays entirely in $[U, V]$; in particular, $\pi_{U V}$ does not contain $W$. Similarly, there exists a path $\pi_{V W}$ in $\Gamma_{\mathcal{D}}$ connecting $V$ and $W$ and staying entirely in $[V, W]$, and a path $\pi_{W U}$ connecting $W$ and $U$ and staying entirely in $[W, U]$. But then the union $\pi_{U V} \cup \pi_{V W} \cup \pi_{W U}$ forms a cycle, which contradicts the assumed acyclicity of $\Gamma_{\mathcal{D}}$. Thus, again, by Proposition A.1 the equivalence (3.1) is satisfied for the domain $\mathcal{D}$.

Note that there do exist connected domains $\mathcal{D}$ and pairwise distinct orders $U, V, W \in \mathcal{D}$ satisfying condition (A.4). Examples are all triples of orders on a common 6-cycle with pairwise distance of two such as, for instance, $a b c, b c a, c a b$ in Figure 2, or $a b c d, b c a d, c a b d$ in Figure 5, etc.

\section{References}

J.M. Abello. The weak Bruhat order of $S_{\Sigma}$, consistent sets, and Catalan numbers. SIAM Journal on Discrete Mathematics, 4(1):1-16, 1991.

J.M. Abello. The majority rule and combinatorial geometry (via the symmetric group). Annales du LAMSADE, 3:1-13, 2004.

K. J. Arrow. Social Choice and Individual Values. Wiley, New York, 1951.

S.P. Avann. Metric ternary distributive semi-lattices. Proc. Amer. Math. Soc., 12, 1961.

H. J. Bandelt and V. Chepoi. A Helly theorem in weakly modular space. Discrete Mathematics, 160:25-39, 1996.

D. Black. On the Rationale of Group Decision-Making. Journal of Political Economy, 56: 23-34, 1948.

C. Chameni-Nembua. Permutoèdre et choix social. Third cycle thesis. Universitè de Paris V, 1989.

\footnotetext{
${ }^{13}$ Note, however, that we cannot use part (i) directly since we do not know yet whether $\mathcal{D}$ is a median domain.
} 
A. Clearwater, C. Puppe, and A. Slinko. Generalizing the single-crossing property on lines and trees to intermediate preferences on median graphs. In Proceedings of the 24th Joint International Conference on Artificial Intelligence (IJCAI-2015), 2015.

Marquis de Condorcet. Essai sur l'application de l'analyse à la probabilité des décisions rendues à la pluralité des voix. Paris, 1785.

V.I. Danilov and G.A. Koshevoy. Maximal Condorcet domains. Order, 30(1):181-194, 2013.

V.I. Danilov, A.V. Karzanov, and G.A. Koshevoy. Condorcet domains of tiling type. Discrete Appl. Math., 160(7-8):933-940, 2012.

G. Demange. Majority relation and median representative ordering. SERIEs, 3(1-2): 95-109, 2012.

E. Elkind, P. Faliszewski, and A. Slinko. Cloning in elections: Finding the possible winners. J. Artif. Intell. Res., 42:529-573, 2011.

E. Elkind, P. Faliszewski, and A. Slinko. Clone structures in voters' preferences. In $A C M$ Conference on Electronic Commerce, EC '12, Valencia, Spain, June 4-8, 2012, pages 496-513, 2012.

P. C. Fishburn. Acyclic sets of linear orders: A progress report. Social Choice and Welfare, 19(2):431-447, 2002.

P.C. Fishburn. Acyclic sets of linear orders. Social Choice and Welfare, 14(1):113-124, 1997.

A. Galambos and V. Reiner. Acyclic sets of linear orders via the bruhat orders. Social Choice and Welfare, 30(2):245-264, 2008.

J.S. Gans and M. Smart. Majority voting with single-crossing preferences. Journal of Public Economics, 59:219-237, 1996.

J.-M. Grandmont. Intermediate Preferences and the Majority Rule. Econometrica, 46: 317-330, 1978.

G. Th. Guilbaud and P. Rosenstiehl. Analyse algébrique d'un scrutin. Mathématiques et Sciences humaines, 4:9-33, 1963.

J. Kemeny. Mathematics without numbers. Daedalus, 88:577-591, 1959.

J. Kemeny and L. Snell. Mathematical Models in the Social Sciences. Ginn, 1960.

S. Klavzar and H.M. Mulder. Median graphs: characterizations, location theory and related structures. Journal of Combinatorial Mathematics and Combinatorial Computing, 30:103-127, 1999. 
F.-C. Kung. Sorting out single-crossing preferences on networks. Social Choice and Welfare, Online first, 2014.

J. Mirrlees. An exploration in the theory of optimal income taxation. Review of Economic Studies, 38:175-208, 1971.

B. Monjardet. Condorcet domains and distributive lattices, 2007. Unpublished lecture notes, Ghent (personal communication).

B. Monjardet. Acyclic domains of linear orders: A survey. In S. J. Brams, W. V. Gehrlein, and F. S. Roberts, editors, The Mathematics of Preference, Choice and Order, Studies in Choice and Welfare, pages 139-160. Springer Berlin Heidelberg, 2009.

H. Moulin. Axioms of Cooperative Decision Making. Cambridge University Press, 1988.

H. M. Mulder. The structure of median graphs. Discrete Math., 24:197-204, 1978.

L. Nebeský. Median graphs. Commentationes Mathematicae Universitatis Carolinae, 12: $317325,1971$.

K. Nehring and C. Puppe. The structure of strategy-proof social choice I: General characterization and possibility results on median spaces. Journal of Economic Theory, 135: 269-305, 2007.

K. Nehring and C. Puppe. Abstract arrowian aggregation. Journal of Economic Theory, 145(2):467 - 494, 2010.

K. Roberts. Voting over income tax schedules. J. Public Economics, 8:329-340, 1977.

P. Rothstein. Representative voter theorems. Public Choice, 72(2-3):193-212, 1991.

A. Saporiti. Strategy-proofness and single-crossing. Theoretical Economics, 4:127-163, 2009.

A. K. Sen. A Possibility Theorem on Majority Decisions. Econometrica, 34:491-499, 1966.

M. van de Vel. Theory of Convex Structures. North-Holland, Amsterdam, 1993.

B. Ward. Majority voting and alternative forms of public enterprises. In J. Margolis, editor, The Public Economy of Urban Communities, pages 112-126, Baltimore, MD, 1965. Johns Hopkins Press. 


\section{Working Paper Series in Economics}

recent issues

No. 92 Clemens Puppe and Arkadii Slinko: Condorcet domains, median graphs and the single-crossing property, June 2016

No. 91 Markus Höchstötter, Mher Safarian, Anna Krumetsadik: Analysis of stochastic technical trading algorithms, June 2016

No. 90 Nikolaus Schweizer and Nora Szech: Optimal revelation of life-changing information, May 2016

No. 89 Helena Barnard, Robin Cowan, Alan Kirman and Moritz Müller: Including excluded groups: The slow racial transformation of the South African university system, May 2016

No. 88 Aniol Llorente-Saguer, Roman M. Sheremeta and Nora Szech: Designing contests between heterogeneous contestants: An experimental study of tie-breaks and bid-caps in all-pay auctions, May 2016

No. 87 Johannes Karl Herrmann and Ivan Savin: Optimal policy Identification: Insights from the German electricity market, March 2016

No. 86 Andranik Tangian: Devaluation of one's labor in labor-commoditiesmoney-commodities-labor exchange as a cause of inequality growth, February 2016

No. 85 Thomas Deckers, Armin Falk, Fabian Kosse and Nora Szech: Homo moralis: Personal characteristics, institutions, and moral decision-making, February 2016

No. 84 Markus Fels: When the affordable has no value, and the valuable is unaffordable: The U.S. market for long-term care insurance and the role of Medicaid, February 2016

No. 83 Uwe Cantner, Ivan Savin, Simone Vannuccini: Replicator dynamics in value chains: Explaining some puzzles of market selection, February 2016

No. 82 Helena Barnard, Robin Cowan, Moritz Müller: On the value of foreign PhDs in the developing world: Training versus selection effects, January 2016

The responsibility for the contents of the working papers rests with the author, not the Institute. Since working papers are of a preliminary nature, it may be useful to contact the author of a particular working paper about results or caveats before referring to, or quoting, a paper. Any comments on working papers should be sent directly to the author. 